\title{
Thermomechanical responses of nonlinear torsional vibration with NiTi shape memory alloy _ alternative stable states and their jumps
}

\author{
Minglu Xia ${ }^{1,2}$, Qingping Sun ${ }^{1 *}$
}

\begin{abstract}
The dynamic response of nonlinear torsional vibration with phase transformable NiTi Shape Memory Alloy (SMA) wire is investigated by experiment in this paper. The thermomechanical responses of NiTi wire as a softening nonlinear damping spring in the torsional vibration system are measured by synchronized acquisition of the rotational angle and temperature under external excitation. Frequency Response Curves (FRCs) at fixed excitation amplitude and Amplitude Response Curves (ARCs) at fixed frequency are obtained in the frequency and amplitude domains respectively. It is found that, as the deformation of NiTi wire goes into the softening nonlinear phase transition region, the smooth and stable dynamic responses along one branch of FRC or ARC will gradually enter into metastable region and eventually become unstable and drastically switch to a new contrasting alternative stable state along the other branch. The jump phenomenon between the alternative stable states on the lower and upper branches of the FRC or ARC and the hysteresis between the jump-up and jump-down are identified in experiments. In addition, the effects of internal and external disturbance (both magnitude and direction) on triggering the jumps between the alternative stable states along the two metastable branches are examined in the time domain. The stability of the nonlinear dynamic response is analyzed by the Duffing oscillator model and interpreted via the stability landscape. For the first time, we directly reveal the alternative stable states and jump phenomena of thermomechanical responses by experiments in the frequency, amplitude and time domains. The results not only showed the important roles of phase transition nonlinearity in bringing multiple equilibrium states and their fast switches, but also provided a solid experimental base for the identification of metastable regions as well as further management of the undesired dynamic responses of vibration system where NiTi is used as a nonlinear damping spring.
\end{abstract}

Keywords: Nonlinear vibration, shape memory alloy, alternative stable state, jump phenomenon, Duffing oscillator, stability. 


\section{Nomenclature}

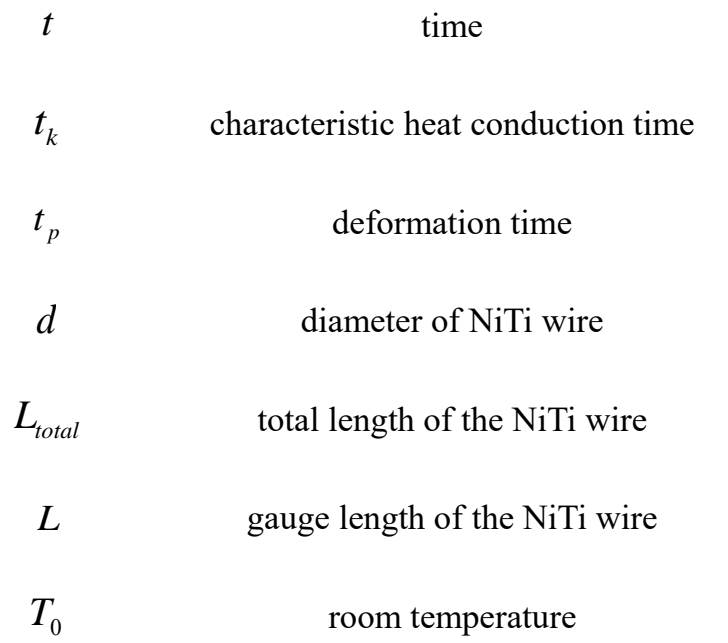

$A_{f}$

$f_{\text {up }}$

$f_{\text {down }}$ average temperature of the NiTi wire

austenite finish temperature

specific heat capacity

thermal conductivity

rotational momentum of inertia

rotation of the NiTi wire in pure torsion

rotation of the NiTi wire in dynamic test

$$
(\theta=\beta-\alpha)
$$

rotation of the mass blocks

external sinusoidal angular excitation

$$
(\alpha=A \sin 2 \pi f t)
$$

external excitation amplitude

external excitation frequency

primary resonance frequency of the

vibration system in the linear elastic region

$$
\text { shear modulus of NiTi }
$$

jump-up frequency

jump-down frequency

$\Delta f \quad$ jump frequency gap $\left(\Delta f=f_{\text {up }}-f_{\text {down }}\right)$

$A_{\text {up }}$

$A_{\text {down }}$

$\Delta A \quad$ jump amplitude gap $\left(\Delta A=A_{u p}-A_{\text {down }}\right)$

$\Delta T$

$\Delta \theta_{u p}$

$\Delta \theta_{\text {down }}$

$\Delta T_{u p}$

$\Delta T_{\text {down }}$

$k_{1}, k_{2}$

$M_{\text {spring }}$

$c_{e q}$

$M_{\text {damper }}$ $\gamma_{s} \quad$ shear strain at the start of phase transition $\gamma_{0}^{P T} \quad$ maximum phase transition shear strain

peak-to-valley magnitude of $\theta$

$$
\text { ( } \theta=\theta_{\max }-\theta_{\min } \text { in one cycle) }
$$

peak-to-valley magnitude of $T$

$$
\text { ( } T=T_{\max }-T_{\min } \text { in one cycle) }
$$

jump-up magnitude of $\Delta \theta$

$$
\left(\Delta \theta_{u p}=\Delta \theta_{u p}^{\text {after }}-\Delta \theta_{u p}^{\text {before }}\right)
$$

jump-down magnitude $\Delta \theta$

$$
\left(\Delta \theta_{\text {down }}=\Delta \theta_{\text {down }}^{\text {before }}-\Delta \theta_{\text {down }}^{\text {after }}\right)
$$

jump-up magnitude $\Delta T$

$$
\left(\Delta T_{u p}=\Delta T_{u p}^{a f t e r}-\Delta T_{u p}^{b e f o r e}\right)
$$

jump-down magnitude $\Delta T$

$$
\left(\Delta T_{\text {down }}=\Delta T_{\text {down }}^{\text {before }}-\Delta T_{\text {down }}^{\text {after }}\right)
$$

spring constants of the effective Duffing oscillator torque of the equivalent nonlinear spring damping coefficient of the equivalent viscous damper torque of the equivalent viscous damper 


\section{Introduction}

Originating from the reversible, first-order martensitic phase transition, NiTi Shape Memory Alloy (SMA) accommodates large recoverable deformation and has large damping capacity (Otsuka and Wayman, 1999). The excellent thermomechanical properties make NiTi an excellent candidate material for vibration control and damping applications (Lagoudas, 2008; Saadat et al., 2002). However, several great challenges still exist in the vibration system with the phase transformable NiTi SMA.

The first challenge lies in the strong thermomechanical coupling in the behavior of NiTi SMA in Figure 1. Two intrinsic internal heat sources are involved in the stress-induced cyclic phase transition of superelastic NiTi: latent heat and hysteresis heat (Shaw and Kyriakides, 1995; Van Humbeeck, 2003; Yin et al., 2014). The generated heat will exchange with the ambient through heat convection and conduction, resulting in a temperature variation of the material. The phase transition stress depends on temperature following the Clausius-Clapeyron relation (Rao and Rao, 1978) and therefore the temperature variation will change the stress-strain relation, which in turn influences the two internal heat sources. Consequently, the stress-strain relation and temperature oscillation are intrinsically coupled with each other. In stress-induced cyclic phase transition, mechanical and thermal aspects of the material should be considered in parallel.

The second challenging issue is the instabilities of dynamic system with NiTi SMA, such as chaos, bifurcation and cusp catastrophe (Nayfeh and Mook, 2008; Schmidt and Tondl., 1986; Stoker, 1966), due to the intrinsic nonlinear and hysteretic behavior of the material in stress-induced phase transition. A typical feature of cusp catastrophe is the jump phenomenon when a nonlinear oscillator is subjected to an external periodic excitation in Figure 2. When increasing the excitation frequency or amplitude, dynamic response ascends on the Frequency Response Curve (FRC) or Amplitude Response Curve (ARC) along 
A-B-C and then jumps up from $\mathrm{C}$ to $\mathrm{D}$. However, when decreasing the frequency or amplitude, the dynamic response takes a different routine and goes by E-D-F and then suddenly jumps down from F to B. Between the jump-up and jump-down, a multi-valued metastable region (B-C and D-F) exists and the dynamic response may overcome the stability barrier and switch from one branch of solution to the alternative metastable branch, giving rise to the jump phenomena (Scheffer et al., 2001; Scheffer and Carpenter, 2003). Specifically, for a vibration system with softening nonlinear NiTi SMA, multiple metastable states of the dynamic response are inevitable and the jumps between these metastable states may occur when the external and internal control parameters fall into certain regions. These regions must be well identified and excluded for vibration system with nonlinear NiTi damping component.

Last but not least, direct experimental observation and systematic investigation on the jump phenomena in the thermomechanical responses of nonlinear dynamic system with NiTi SMA require careful design of the testing platform and large quantity of experimental data. In modeling and simulation, there has been a lot of work over the past two decades. The softening nonlinear features and jumps of thermomechanical responses (Bernardini and Rega, 2005; Lagoudas et al., 2005; Machado and Lagoudas, 2006; Masuda and Noori, 2002; Piccirillo et al., 2010a, b; Seelecke, 2002), the softening-hardening nonlinear features (Bernardini and Vestroni, 2003; Lacarbonara et al., 2004; Masuda and Noori, 2002) and effects of system parameters on the jump phenomenon (Bernardini and Rega, 2010; Bernardini and Vestroni, 2003; Lacarbonara et al., 2004; Lagoudas et al., 2005; Machado and Lagoudas, 2006; Masuda and Noori, 2002; Seelecke, 2002) have been well identified. In addition to jump phenomenon, chaos and bifurcation (Bernardini and Rega, 2005; Dos Santos and Savi, 2009; Lacarbonara et al., 2004; Machado et al., 2007a; Machado et al., 2007b; Machado et al., 2003, 2004; Piccirillo et al., 2008, 2009a, b; Savi, 2015; Savi and 
Nogueira, 2010; Savi and Pacheco, 2002; Savi et al., 2002) were also studied in softening nonlinear dynamic systems with NiTi SMA. In sharp contrast, much less has been achieved on the experimental aspect. Some early experimental works (Collet et al., 2001; Feng and Li, 1996; Graesser, 1995; Lammering and Schmidt, 2001; Li and Feng, 1997; Schmidt and Lammering, 2004a, b) have observed the softening nonlinear features of the transmissibility curve. The resonance frequencies and peak responses strongly depend on the external excitations. The jump of rotation on the FRC in torsional vibration of NiTi wire was directly identified for the first time in 2012 (Doaré et al., 2012; Moussa et al., 2012). The simultaneous jumps of rotation and temperature were further quantified for a mass-NiTi wire torsional vibration system in the frequency domain (Xia and Sun, 2015). However, the existing works are limited to the frequency domain only and there has been no report on the thermomechanical responses in the amplitude and time domain remains. In particular, there is a lack of systematic experimental investigation on the jumps in the frequency, amplitude and time domains, which impedes a comprehensive understanding of the nonlinear phenomenon and prevents the NiTi SMA from further applications.

This paper aims at identifying the alternative stable states and the jump phenomena of dynamic responses in the frequency, amplitude and time domains for a nonlinear torsional vibration system with NiTi wire. Basic thermomechanical properties of the material and the testing platform are introduced in Section 2. The alternative stable states and the jumps of thermomechanical responses in the frequency, amplitude and time domains are reported and discussed in Section 3. The summary and key conclusions are given in Section 4.

\section{Material, sample preparation and experimental setup}

A polycrystalline superelastic Ni-49.6at $\% \mathrm{Ti}$ SMA wire (diameter $d=1.63 \mathrm{~mm}$, total 
length $L_{\text {total }}=60.00 \mathrm{~mm}$ and gauge length $L=20.00 \mathrm{~mm}$ from Nitinol, USA) is used as a nonlinear damping spring in the torsional vibration system in Figure 3. Basic mechanical and thermal properties of the NiTi wire are listed in Table 1. Room temperature $\left(T_{0}\right)$ is maintained at $T_{0} \approx 25^{\circ} \mathrm{C}$ during the experiments and therefore superelasticity of the specimen can be guaranteed. As a long cylindrical shaft under torsion, the NiTi wire will first experience martensitic phase transition on the cylindrical surface at the rotation of $\varphi=\frac{2 L \gamma_{s}}{d} \approx 15.47^{\circ}$ ( $\gamma_{s}$ is the shear strain at the start of phase transition). Using the shear modulus of austenite phase $(G=16.5 \mathrm{GPa})$ and rotational momentum of inertia $\left(J=0.032 \mathrm{~kg} \cdot \mathrm{m}^{2}\right)$ of the torsional vibration system, the primary resonance frequency in the linear elastic region is estimated as $f_{0}=\frac{1}{2 \pi} \sqrt{\frac{\pi G d^{4} / 32 L}{J}} \approx 0.673 \mathrm{~Hz}$.

Before the torsional vibration tests, the NiTi wire was trained by two-way cyclic torsion under quasi-isothermal conditions $(f=0.001 \mathrm{~Hz})$ for 200 cycles in Figure 4(a) to ensure a repeatable torque-angle relation. The measured torque-angle relations $(M=M(\varphi, f))$ at $1^{\text {st }}$, $50^{\text {th }}, 100^{\text {th }}, 150^{\text {th }}$ and $200^{\text {th }}$ cycle are examined in particular. It is seen that the $M \sim \varphi$ relation become completely repeatable after $150^{\text {th }}$ cycle (in red). After training, $M \sim \varphi$ relation of the NiTi wire was characterized at $\varphi= \pm 100^{\circ}$ and different frequencies of $f=0.001 / 0.35 / 0.45 / 0.55 / 0.65 / 0.75 \mathrm{~Hz}$. The steady state responses are summarized in Figure 4(b) and it is seen that the non-isothermal $M \sim \varphi$ relations significantly deviate from the isothermal case ( $f=0.001 \mathrm{~Hz}$ denoted by dashed line) in pure torsion. However, in the frequency range of $f \in[0.35 \mathrm{~Hz}, 0.75 \mathrm{~Hz}]$, which is the focus of this paper, the differences among these steady state non-isothermal $M \sim \varphi$ relations are negligible and the $M \sim \varphi$ relations can be represented by the same curve. At an intermediate frequency of $f=0.60 \mathrm{~Hz}$, 
pure torsion are performed at $\varphi= \pm 10^{\circ} / \pm 20^{\circ} / \pm 30^{\circ} / \pm 40^{\circ} / \pm 50^{\circ} / \pm 60^{\circ} / \pm 70^{\circ} / \pm 80^{\circ} / \pm 90^{\circ}$ $/ \pm 100^{\circ}$ and all of the steady state $M \sim \varphi$ relations collapse on a single curve in Figure 4(b). It is noticed that due to the internal elastic core inside the NiTi wire during torsion, the rotational angle cannot be totally recovered at $M=0$ and there is a very small residual rotation $\left(1^{\circ} \sim 2^{\circ}\right)$. In order to check if there is any cyclic plasticity of the NiTi wire during vibration tests, each specimen was examined after the dynamic tests by pure torsion at $f=0.60 \mathrm{~Hz}$ and $\varphi= \pm 100^{\circ}$. It's seen from Figure 4(c) that degradation of the NiTi wires during the torsional vibration tests is indeed ignorable.

As shown in Figure 3, the dynamic testing platform consists of the following four parts. (1) An electric rotational stepping motor on the electromagnetic test instrument (ElectroForce 3510 from BOSE, USA) to provide external sinusoidal angular excitation ( $\alpha=A \sin 2 \pi f t$ ). (2) Specimen clamping with special designed teeth to avoid possible sliding. (3) A beam with two mass blocks attached at its two ends to give rotational momentum of inertia $\left(J=0.032 \mathrm{~kg} \cdot \mathrm{m}^{2}\right)$ at the upper end of the NiTi wire. The beam is held by a single direction thrust ball bearing so as to rotate freely without axial force. (4) Synchronized data acquisition of the mechanical and thermal responses. The relative rotational angle of the NiTi wire $(\theta(t)=\beta(t)-\alpha(t))$ is obtained from the external excitation $(\alpha(t))$ and rotation of beam $(\beta(t))$ with a rotary motion sensor (PS-2120A from PASCO, USA). Volume average temperature of the NiTi wire $\left(T=\frac{1}{V} \int T(x, t) d x\right)$, as the thermal response of the dynamic system, is measured by an infrared camera (SC7700M from FLIR, USA). The NiTi wire is painted with matt black paint (TS-6 from Tamiya, Japan) to enhance the emissivity for thermal imaging. (Remark: in the testing frequency range of $f \in[0.4 \mathrm{~Hz}, 0.7 \mathrm{~Hz}]$, the characteristic heat conduction time $t_{k}=\lambda d^{2} / 4 k \approx 0.11 s$ over the circular cross-section of the NiTi wire is much shorter than the 
characteristic deformation time $t_{p}=1 / f \in[1.43 \mathrm{~s}, 2.50 \mathrm{~s}]$. So the temperature field over the cross-section can be regarded as uniform and is equal to the temperature at the surface.)

To validate the dynamic testing platform, torsional vibration at the excitation of $A=12^{\circ}$ and $f=0.580 \mathrm{~Hz}$ is taken as an example for analysis. From the simultaneously monitored axial displacement and axial force in Figure 5(a), it is seen that the maximum compression force on the specimen is $-4 \mathrm{~N}$ (equivalent to a compressive stress of $1.92 \mathrm{MPa}$ ), far below the elastic buckling load of $-63.24 \mathrm{~N}$ (Timoshenko and Goodier, 1969). The maximum tensile stress is $0.48 \mathrm{MPa}$ and maximum tensile strain is $0.15 \%$. The axial stress and axial strain are negligible compared with the applied shear stress and shear strain. Consequently, the torsional vibration tests with ignorable axial stress and axial strain can be guaranteed. In the evolution of thermomechanical responses in Figure 5(b), both the rotational angle $\theta(t)$ and temperature $T(t)$ experience a transient stage and a steady stage under external excitation and a free damping stage after the excitation is off. $\theta(t)$ oscillates symmetrically around $0^{\circ}$ but $T(t)$ is non-symmetric since the mean temperature increases in the transient stage due to the accumulation of hysteresis heat and heat exchange with the ambient (Yin et al., 2014). Figure 5(c) shows the synchronized measurements of $\alpha(t), \beta(t), \theta(t)$ and $T(t)$ in the initial transient stage $(0 \sim 10 \mathrm{~s})$. It is seen that the oscillation period of $T(t)$ is only half of that for $\theta(t)$ due to the two phase transition cycles in one excitation cycle. Figure 5(d) shows the oscillations of $\theta(t)$ and $T(t)$ in the steady stage $(60 \mathrm{~s} \sim 65 \mathrm{~s})$. Referring to the inserted $M \sim \varphi$ relation of the NiTi wire in pure torsion, we see that it's the hysteresis heat accumulation and latent heat release/absorption during phase transition that leads to a mean temperature rise in the transient stage and temperature oscillation in the steady stage. 


\section{Experimental results and discussions}

\subsection{Alternative stable states and their jumps in the frequency domain}

Under a given external excitation amplitude and frequency, the steady-state peak-to-valley magnitudes of rotational angle and temperature ( $\Delta \theta=\theta_{\max }-\theta_{\min }$ and $\Delta T=T_{\max }-T_{\min }$ in one cycle) are quantified as the mechanical and thermal responses of the nonlinear vibration system. To capture the alternative stable states and the consequent jumps of $\Delta \theta$ and $\Delta T$ in the frequency domain under given excitation amplitude $A$, the values of applied excitation frequency $f$ should well span the primary resonance frequency $f_{0}$. In the experiment, for each of the five excitation amplitudes $\left(A=1.5^{\circ}, 3^{\circ}, 6^{\circ}, 9^{\circ}\right.$ and $\left.12^{\circ}\right)$, a total of 61 different excitation frequencies are selected in the range of $f \in[0.400 \mathrm{~Hz}, 0.700 \mathrm{~Hz}]$ around $f_{0}$. Independent experiment was performed at each frequency according to ascending order (from $0.400 \mathrm{~Hz}$ up to $0.700 \mathrm{~Hz}$ at $0.005 \mathrm{~Hz}$ interval) to search for the jump-up phenomena and descending order (from $0.700 \mathrm{~Hz}$ down to $0.400 \mathrm{~Hz}$ at $0.005 \mathrm{~Hz}$ interval) to search for the jump-down phenomena respectively. The obtained FRCs of $\Delta \theta$ and $\Delta T$ are summarized on the left side of Figure 6.

It is seen that under small excitation amplitude (e.g., $A=1.5^{\circ}$ in black), the deformation of NiTi wire is still in the linear elastic range and the $\Delta T$ is negligible. As the excitation amplitude gradually increases (e.g., $A=3^{\circ}$ in red), $\Delta \theta$ and $\Delta T$ become larger as the NiTi wire enters phase transition region and significant asymmetries appear in the FRCs. At the frequency of $0.625 \mathrm{~Hz}$, the FRCs become steep, implying the jump and drop in the FRCs of $\Delta \theta$ and $\Delta T$. The difference of the FRCs following ascending and descending frequency orders becomes distinct. At larger excitation amplitude (e.g., $A=6^{\circ}$ in green), the jump-up of $\Delta \theta$ following ascending frequency order and jump-down of $\Delta \theta$ following descending frequency order are eventually captured at $f_{\text {up }}=0.580 \mathrm{~Hz}$ and $f_{\text {down }}=0.570 \mathrm{~Hz}$ 
respectively in the experiments. Simultaneous jump-up and jump-down of $\Delta T$ at exactly the same $f_{\text {up }}$ and $f_{\text {down }}$ are observed on the FRC of temperature response in Figure 6(b). When further increasing the excitation amplitudes (e.g., $A=9^{\circ}$ or $12^{\circ}$ in blue or cyan), the jumps of $\Delta \theta$ and $\Delta T$ in the frequency domain become more significant.

The jump frequencies ( $f_{\text {up }}$ and $\left.f_{\text {down }}\right)$, jump frequency gap $\left(\Delta f=f_{\text {up }}-f_{\text {down }}\right)$ and jump magnitudes of thermomechanical response $\left(\Delta \theta_{u p}\right.$ and $\Delta \theta_{\text {down }}, \Delta T_{u p}$ and $\Delta T_{\text {down }}$ ) are summarized in Figure 7. It is seen that both $f_{\text {up }}$ and $f_{\text {down }}$ are very close to but lower than the estimated primary resonance frequency $\left(f_{0} \approx 0.673 \mathrm{~Hz}\right)$. As the excitation amplitude $A$ increases, both $f_{u p}$ and $f_{\text {down }}$ decrease but the frequency gap $\Delta f$ increases. At the same time, the jump magnitudes ( $\Delta \theta_{u p}$ and $\Delta \theta_{\text {down }}, \Delta T_{u p}$ and $\Delta T_{\text {down }}$ ) keep increasing with $A$.

To interpret the above jump phenomena, an effective Duffing oscillation model and a lumped heat transfer analysis (Xia and Sun, 2015) are proposed to quantify the mechanical and thermal responses of the nonlinear torsional vibration system with NiTi wire. With appropriate simplification and approximation, the phase transition nonlinearity and dissipative property of the NiTi wire in torsion are separately modelled as an equivalent cubic nonlinear spring $\left(M_{\text {spring }}=k_{1} \theta+k_{2} \theta^{3}\right)$ and an equivalent angular viscous damper $\left(M_{\text {damper }}=c_{e q} \dot{\theta}\right)$ as shown in Figure 8(a).

The equation of motion in Figure 3 can be expressed as,

$$
J \ddot{\beta}=-c_{e q} \dot{\theta}-k_{1} \theta-k_{2} \theta^{3}
$$

Replacing $\beta$ by $\theta$ and $\alpha(\beta=\theta+\alpha=\theta+A \sin 2 \pi f t)$, we have

$$
J \ddot{\theta}+c_{e q} \dot{\theta}+k_{1} \theta+k_{2} \theta^{3}=4 A J \pi^{2} f^{2} \sin 2 \pi f t
$$

Assuming that the steady state solution is a sinusoidal periodic function with frequency $f$ at phase angle $\chi$, 


$$
\theta=\frac{\Delta \theta}{2} \cos (2 \pi f t-\chi)
$$

The steady state frequency response is obtained with the method of multiple scales

$$
\left[\left(k_{1}-4 \pi^{2} f^{2} J\right) \frac{\Delta \theta}{2}+\frac{3}{4} k_{2}\left(\frac{\Delta \theta}{2}\right)^{3}\right]^{2}+\left(\pi f c_{e q} \Delta \theta\right)^{2}=\left(4 A J \pi^{2} f^{2}\right)^{2}
$$

Meanwhile, the "backbone" curve in the frequency domain is given by

$$
\Delta \theta=4 \sqrt{\frac{4 \pi^{2} f^{2} J-k_{1}}{3 k_{2}}}
$$

Assuming the shear behavior of NiTi wire under partial phase transition in torsion as ideal elastoplastic type with perfect plasticity in Figure 8(b), a lumped heat transfer analysis is performed to establish the relation between the temperature oscillation $(\Delta T)$ and the rotation $(\Delta \theta)$ of the NiTi wire,

$$
\Delta T=\left\{\begin{array}{cc}
0 & \left(\Delta \theta \leq \frac{4 L \gamma_{s}}{d}\right) \\
{\left[\frac{d}{6 L \gamma_{0}^{P T}} \Delta \theta+\frac{16 L^{2} \gamma_{s}^{3}}{3 d^{2} \gamma_{0}^{P T}} \frac{1}{(\Delta \theta)^{2}}-\frac{\gamma_{s}}{\gamma_{0}^{P T}}\right] \frac{l_{0}}{\lambda}} & \left(\Delta \theta>\frac{4 L \gamma_{s}}{d}\right)
\end{array}\right.
$$

In summary, Eq. (1) is the governing equation of the nonlinear dynamic system under external sinusoidal excitation. Eq. (2) is an implicit solution of rotational angle amplitude $(\Delta \theta)$ as a function of the external excitation ( $f$ and $A$ ). Eq. (3) determines the "backbone" curve of FRCs in the frequency domain. Eq. (4) gives the temperature oscillation $(\Delta T)$ at a given rotational angle ( $\Delta \theta)$. Eq. (2) and Eq. (4) are the approximate solutions of the thermomechanical responses in both frequency and amplitude domains.

Using the approaches proposed in (Xia and Sun, 2015), the effective damping coefficient and equivalent spring constants are independently quantified as: $c_{e q}=0.0052 \mathrm{~N} \cdot \mathrm{m} \cdot \mathrm{s}$, $k_{1}=0.480 \mathrm{~N} \cdot \mathrm{m}$ and $k_{2}=-0.105 \mathrm{~N} \cdot \mathrm{m}$. Together with the basic material properties in Table 1, the FRCs of the mechanical and thermal responses are obtained from the above theoretical analysis and plotted on the right side of Figure 6 and Figure 7 for comparison. 
It is seen that the FRCs strongly bend to the left, showing typical softening nonlinear properties. As the excitation amplitude $A$ increases, the magnitudes of thermomechanical response grow larger and the jump frequencies ( $f_{u p}$ and $\left.f_{\text {down }}\right)$ consistently shift to the left. At the same time, the frequency gap $(\Delta f)$ and jump magnitudes $\left(\Delta \theta_{u p}\right.$ and $\Delta T_{u p}, \Delta \theta_{\text {down }}$ and $\left.\Delta T_{\text {down }}\right)$ keep increasing with the excitation amplitude. We see that the general features of the FRCs from the experiments and modeling agree well with each other. Although there are some quantitative errors, the thermomechanical responses of a nonlinear torsional vibration system with NiTi wire in the frequency domains can be well quantified by the simplified Duffing oscillation model and lumped heat analysis.

\subsection{Alternative stable states and their jumps in the amplitude domain}

In the amplitude domain, to search for the alternative stable states and capture their jumps, the excitation frequency $f$ is kept as a constant while the excitation amplitude $A$ is slowly changed following ascending and descending orders respectively. In the experiments, five excitation frequencies ( $f=0.50 \mathrm{~Hz}, 0.52 \mathrm{~Hz}, 0.54 \mathrm{~Hz}, 0.56 \mathrm{~Hz}$ and $0.58 \mathrm{~Hz}$ ) were selected to perform the torsional vibration tests. At each frequency, A was taken from the range of $A \in\left[6.0^{\circ}, 12.0^{\circ}\right]$ and a total of 122 independent experiments were performed from $6.0^{\circ}$ up to $12.0^{\circ}$ and from $12.0^{\circ}$ down to $6.0^{\circ}$ with $0.1^{\circ}$ interval. The steady state $\Delta \theta$ and $\Delta T$ are measured and plotted against $A$ as ARCs on the left side of Figure 9.

Different from the FRCs in Section 3.1, the ARCs do not have a peak response in the intermediate region and both $\Delta \theta$ and $\Delta T$ monotonically increase with $A$ at each excitation frequency. At a small excitation frequency (e.g., $f=0.50 \mathrm{~Hz}$ in black), the deformation of NiTi wire is within the linear elastic region of the material. No jump phenomena are observed in the testing amplitude range. When $f$ is increased up to $0.52 \mathrm{~Hz}$ in red, simultaneous jump-up of $\Delta \theta$ and $\Delta T$ are observed on the ARCs at $A_{u p}=11.5^{\circ}$ 
following ascending order and simultaneous jump-down occurs at $A_{\text {down }}=10.2^{\circ}$ following descending order, thus bringing a multi-valued region $\left(\Delta A=A_{u p}-A_{\text {down }}\right)$ in the amplitude domain. As $f$ is further increased to $0.54 \mathrm{~Hz}$ and $0.56 \mathrm{~Hz}$ (in green and blue respectively), the lower branch will drift up while the upper branch will drift down. Consequently the jump magnitudes of both rotational angle $\left(\Delta \theta_{u p}\right.$ and $\left.\Delta \theta_{\text {down }}\right)$ and temperature oscillation $\left(\Delta T_{u p}\right.$ and $\left.\Delta T_{\text {down }}\right)$ will decrease with $f$. At the same time, the jump amplitudes $\left(A_{\text {up }}\right.$ and $\left.A_{\text {down }}\right)$ shift to the left with the increase of excitation frequency. At very large frequency (e.g., $f=0.58 \mathrm{~Hz}$ in cyan), the entire ARCs are on the upper branch without jump phenomena in the testing amplitude range, though the they may be observed at smaller amplitude.

To quantify the ARCs of thermomechanical responses in the amplitude domain, Eq. (2) and Eq. (4) are used with the same parameters as Section 3.1 and the theoretical modeling results are shown on the right side of Figure 9 and Figure 10. It is seen that the nonlinear features of experimental data are well predicted by the theoretical modeling results. Jump-up and jump-down are captured on the ARCs following ascending and descending amplitude paths in the amplitude domain. The modeling results also confirm that the jump amplitudes $\left(A_{\text {up }}\right.$ and $\left.A_{\text {down }}\right)$, jump amplitude gap $(\Delta A)$ and jump magnitudes $\left(\Delta \theta_{u p}\right.$ and $\Delta \theta_{\text {down }}, \Delta T_{u p}$ and $\Delta T_{\text {down }}$ ) decrease with the excitation frequency $(f)$ in the testing frequency range.

\subsection{Alternative stable states and their jumps in the time domain}

In the frequency and amplitude domains, the existence of alternative stable states only manifests as separated branches of solutions and the consequent jumps between them on the FRCs and ARCs. To explore how the transition or jump phenomenon takes place, we have to move to the time domain. The multi-valued region between jump-down and jump-up in the frequency and amplitude domains are actually metastable and prone to change when subject to disturbance. When the external excitation frequency or amplitude falls into such metastable 
region, depending on the magnitude and direction of external disturbance, the metastable state of the dynamic responses may quickly shift to an alternative one in the time domain, illustrating the instability nature of the nonlinear vibration system.

In this section, the torsional vibration tests are performed to capture the jump phenomena in the time domain. To search for the critical external excitation, the excitation amplitude is gradually reduced to narrow the frequency gap $(\Delta f)$ between jump-up and jump-down in the frequency domain (see Section 3.1). When $A=3^{\circ}$, the jump-up and jump-down almost overlap $(\Delta f \approx 0)$ and then the excitation frequency is changed slowly around the jump frequency to further identify the critical excitation frequency.

Under the excitation of $A=3^{\circ}$ and $f=0.62160 \mathrm{~Hz}, 0.62170 \mathrm{~Hz}, 0.62180 \mathrm{~Hz}$, the evolutions of $\theta(t)$ and $T(t)$ are plotted in Figure 11. It is seen at $f=0.62160 \mathrm{~Hz}$ the small steady state $\Delta \theta$ and $\Delta T$ are typical of the dynamic responses on the lower branch of the FRC or ARC when $f<f_{\text {down }}$ or $A<A_{\text {down }}$. On the other hand, at $f=0.62180 \mathrm{~Hz}$ in Figure 11(b), the system response reaches a different steady state with large oscillation magnitude, which is typical of the response on the upper branch when $f>f_{u p}$ or $A>A_{u p}$. Different from the above two cases, at the critical excitation of $A=3^{\circ}$ and $f=0.62170 \mathrm{~Hz}$, evolutions of the thermomechanical responses experience a spontaneous transition from the lower branch to the alternative upper branch in the time domain. After the initial transient state, the dynamic responses first settle down to the metastable state I, very similar to the lower branch in Figure 11(a). After 150s, the dynamic response gradually but spontaneously shifts to a metastable state II, which is close to the upper branch response with large oscillation magnitude in Figure 11(b). In this way, we captured the spontaneous jump phenomena of thermomechanical responses from the lower branch of solution to the alternative upper branch in the time domain in one single experiment. When further enlarging 
and examining $\theta(t)$ and $T(t)$ of the two metastable states in Figure 12, the phase angle change of the dynamic responses becomes very distinct. In metastable state I, rotation of the specimen is phase lagging referring to the external excitation. However, in metastable state II, the mechanical response is leading the phase. In the new transient state between these two metastable states, the thermomechanical responses experience phase inversion and this is a key characteristic of the jump phenomena.

The above spontaneous jump phenomena from the lower branch to the alternative upper branch are due to the internal intrinsic disturbances (e.g., friction, temperature fluctuation, etc.). The influence of excitation and disturbance on the jump phenomena in the time domain will be further investigated by introducing the external disturbance by hand in the following. Positive disturbance is defined as pushing forward the mass blocks to enlarge the rotational angle while negative disturbance pulls them back to reduce the rotation during the steady state oscillation.

At $A=9^{\circ}$, torsional vibration tests are performed at $f=0.450 \mathrm{~Hz}, 0.600 \mathrm{~Hz}$ and $0.531 \mathrm{~Hz}$, corresponding to the stable lower branch, stable upper branch and metastable region respectively in the frequency domain in Figure 6. At $f=0.450 \mathrm{~Hz}$ and $0.600 \mathrm{~Hz}$, a negative and a positive disturbance are exerted at about 100s and 200s respectively and the thermomechanical responses are shown in Figure 13(a) and (b). It is seen that at these two frequencies away from the metastable region in the frequency domain, neither a positive nor negative disturbance can induce the jump phenomena in the time domain. This is because at excitations in the stable region, only one stable focus exists and therefore there is only one stable solution. At $f=0.531 \mathrm{~Hz}$ within the metastable region, a positive and a negative disturbance were applied at about 100 s in the steady state in two independent experiments in Figure 13(c). It is seen that the new transient state induced by the negative disturbance leads to the jump phenomena while the new transient state after a positive disturbance quickly dies 
away without jumps. Such strong direction dependence of the jump phenomena can be qualitatively interpreted by state plane as shown on the right side of Figure 13, where it is clearly seen that different external disturbance can drag the steady state response towards different directions. A negative disturbance tends to move the dynamic response towards the upper branch stable focus and even go across the boundary if the disturbance is large enough, thus bringing about a state shift from the lower branch of solution to the alternative upper branch. On the other hand, a positive disturbance drags the steady state response towards the lower branch stable focus further away from the saddle point, therefore always staying on the lower branch without jumps to the alternative upper branch in the time domain.

Similar influences of the external disturbance on the jump phenomena are also observed when the excitation is within the metastable region in the amplitude domain at $f=0.56 \mathrm{~Hz}$ in Figure 9. The dynamic responses are stable at $A=6.0^{\circ}$ on the lower branch and $A=8.0^{\circ}$ on the upper branch but metastable at $A=6.6^{\circ}$ in the amplitude domain. At $A=6.0^{\circ}$ and $8.0^{\circ}$, there are always no jumps in the time domain regardless of external disturbances in Figure 14(a) and (b). At $A=6.6^{\circ}$, a negative disturbance can induce the jump phenomena in Figure 14(c). However, the jumps do not occur after a positive disturbance at the same external excitation. It is seen that the jump phenomena in the time domain are significantly affected by the excitation and external disturbance. When the external excitation is in the stable (or single-valued) region, there are always no jump phenomena regardless of external disturbance. At excitations within the metastable (or multi-valued) region, a positive disturbance cannot induce such jumps as well. Only a negative external disturbance under the external excitations within the metastable region in the frequency or amplitude domain can trigger the jump phenomena of thermomechanical responses in the time domain.

To further investigate the effects of external disturbance on the jumps, nonlinear dynamic tests are performed in the metastable region with small and large external disturbances. At 
$A=9^{\circ}$ and $f=0.530 \mathrm{~Hz}$, the system is metastable in the frequency domain in Figure 6 . With small negative disturbance at 100 s and large negative disturbance at 200 s during the steady state oscillation, evolutions of the thermomechanical responses are shown in Figure 15(a). It is seen that the new transient state induced by small disturbance dies away after some time while the large negative disturbance triggers the jump phenomena in the time domain. This may be because the large negative disturbance provides enough energy to overcome the barrier and go across the boundary to reach the upper branch stable focus in the state plane. If the exerted disturbances are positive as shown in Figure 15(b), neither a small nor a large disturbance can induce the jump phenomena and this is consistent with the previous results. At the excitation of $A=9.2^{\circ}$ and $f=0.54 \mathrm{~Hz}$, within the metastable region in the amplitude domain in Figure 9, similar results are also observed as shown in Figure 16. The large negative disturbance can induce the jump phenomena in the time domain while a small negative disturbance and positive disturbance (no matter small or large) can only lead to a new transient state which dies away quickly without the jump phenomena in the time domain. It is seen that the jumps are also inherently associated with the disturbance and only a proper external disturbance (both in direction and magnitude) can trigger the jump phenomena of thermomechanical responses in the time domain.

The multi-values of FRC and ARC or alternative stable states of the nonlinear vibration system as well as their jumps are summarized in terms of stability landscape in Figure 17, where the stability of steady state dynamic response depends on both external excitation and response. When the excitations fall into the stable region, the dynamic response is single-valued, corresponding to only one minimum on the stability curve and there are always no jump phenomena regardless of disturbance. When the excitation is within the metastable region, there are two minima on the stability curve, implying two metastable states on the 
lower and upper branches respectively. The external disturbance can help the system to overcome the barrier to reach an energetically more favorable alternative metastable state, bringing about the jump phenomena in the time domain. At the critical conditions, the jump phenomena will occur spontaneously without observable external disturbance.

\section{Summary and conclusions}

In this paper, experimental investigations of the dynamic responses of a nonlinear vibration system with phase transformable NiTi SMA are performed for the first time in the frequency, amplitude and time domains. Alternative stable states and simultaneous jump phenomena of thermomechanical responses are studied on both FRCs and ARCs. The fast transition process between the two metastable states is captured and the effects of disturbance on triggering the jumps are examined in the time domain. Systematic data of the metastable region where jump phenomena may happen are well identified by experiments. The main conclusions of this paper are the followings:

- Degree of the nonlinearity and damping capacity of the NiTi SMA spring during phase transition play a critical role on the stability of the nonlinear dynamic responses. By changing the amount of phase transition of the NiTi wire, the external excitation amplitude $A$ can significantly influence the stability of dynamic responses. As $A$ increases, both lower and upper branches of the FRCs drift up and $f_{\text {up }}$ and $f_{\text {down }}$ decrease, but $\Delta f, \Delta \theta_{\text {up }}, \Delta \theta_{\text {down }}, \Delta T_{\text {up }}, \Delta T_{\text {down }}$ increase.

- Simultaneous jump phenomena of mechanical response and thermal response exist on the ARC in the amplitude domain for nonlinear vibration with NiTi SMA. As excitation frequency $f$ increases, the lower branch of the ARCs will drift up while the upper branch will drift down. The $A_{u p}, A_{\text {down }}, \Delta A$ and $\Delta \theta_{u p}, \Delta \theta_{\text {down }}, \Delta T_{\text {up }}, \Delta T_{\text {down }}$ keep 
decreasing with $f$.

- Jump phenomena between the alternative stable states are inherently associated with disturbance. They can either occur spontaneously at critical excitations without observable external interference or occur under external disturbance of appropriate direction and magnitude. Based on the experiments in the three domains, alternative stable states can be accurately identified and the stability landscape is constructed to interpret the occurrence of jump phenomena and to manage the vibration system. Examples include promoting the desired dynamic responses and preventing the undesired ones.

\section{Acknowledgements}

The financial support from the Hong Kong Research Grants Council through GRF Project No. 619113, 16209414, N_HKUST 617/14 and the 973 Program of China through Project No. 2014CB046902 is gratefully acknowledged. Technical assistance from the Advanced Engineering Material Facility (AEMF), Materials Characterization and Preparation Facility (MCPF) and Design and Manufacturing Services Facility (DMSF) of the Hong Kong University of Science and Technology is highly appreciated.

\section{References}

Bernardini, D., Rega, G., 2005. Thermomechanical modelling, nonlinear dynamics and chaos in shape memory oscillators. Math. Comput. Model. Dyn. Syst. 11, 291-314.

Bernardini, D., Rega, G., 2010. The influence of model parameters and of the thermomechanical coupling on the behavior of shape memory devices. Int. J. Non-linear Mech. 45, 933-946.

Bernardini, D., Vestroni, F., 2003. Non-isothermal oscillations of pseudoelastic devices. Int. J. Non-linear Mech. 38, 1297-1313.

Collet, M., Foltete, E., Lexcellent, C., 2001. Analysis of the behavior of a Shape Memory Alloy beam under dynamical loading. Eur J Mech a-Solid 20, 615-630. 
Doaré, O., Sbarra, A., Touzé, C., Ould Moussa, M., Moumni, Z., 2012. Experimental analysis of the quasi-static and dynamic torsional behaviour of shape memory alloys. Int. J. Solids Struct. 49, 32-42.

Dos Santos, B.C., Savi, M.A., 2009. Nonlinear dynamics of a nonsmooth shape memory alloy oscillator. Chaos, Solitons and Fractals 40, 197-209.

Feng, Z.C., Li, D.Z., 1996. Dynamics of a mechanical system with a shape memory alloy bar. J. Intell. Mater. Syst. Struct. 7, 399-410.

Graesser, E.J., 1995. Effect of intrinsic damping on vibration transmissibility of nickel-titanium shape memory alloy springs. Metallurgical and Materials Transactions A-Physical Metallurgy and Materials Science 26, 2791-2796.

Lacarbonara, W., Bernardini, D., Vestroni, F., 2004. Nonlinear thermomechanical oscillations of shape-memory devices. Int. J. Solids Struct. 41, 1209-1234.

Lagoudas, D.C., 2008. Shape Memory Alloys: Modeling and Engineering Applications. Springer.

Lagoudas, D.C., Machado, L.G., Lagoudas, M., 2005. Nonlinear vibration of a one-degree of freedom shape memory alloy oscillator: A numerical-experimental investigation, Proceedings of the 46th AIAA/ASME/ASCE/AHS/ASC Structures, Structural Dynamics and Materials Conference.

Lammering, R., Schmidt, I., 2001. Experimental investigations on the damping capacity of NiTi components. Smart Mater. Struct. 10, 853-859.

Li, D.Z., Feng, Z.C., 1997. Dynamic properties of pseudoelastic shape memory alloys, Proc. SPIE 3041, Smart Structures and Materials 1997: Smart Structures and Integrated Systems.

Machado, L.G., Lagoudas, D.C., 2006. Nonlinear dynamics of a SMA passive vibration damping device, Proc. SPIE 6169, Smart Structures and Materials 2006: Damping and Isolation.

Machado, L.G., Lagoudas, D.C., Savi, M.A., 2007a. Isothermal and non-isothermal oscillations of a pseudoelastic oscillator: Lyapunov exponents estimation, Proceedings of COBEM 2007, 19th International Congress of Mechanical Engineering.

Machado, L.G., Lagoudas, D.C., Savi, M.A., 2007b. Nonlinear dynamics and chaos in a shape memory alloy pseudoelastic oscillator, Proc. SPIE 6525, Active and Passive Smart Structures and Integrated Systems 2007.

Machado, L.G., Savi, M.A., Pacheco, P.M.C.L., 2003. Nonlinear dynamics and chaos in coupled shape memory oscillators. Int. J. Solids Struct. 40, 5139-5156.

Machado, L.G., Savi, M.A., Pacheco, P.M.C.L., 2004. Bifurcations and crises in a shape memory oscillator. Shock and Vibration 11, 67-80.

Masuda, A., Noori, M., 2002. Optimization of hysteretic characteristics of damping devices based on pseudoelastic shape memory alloys. Int. J. Non-linear Mech. 37, 1375-1386.

Moussa, M.O., Moumni, Z., Doare, O., Touze, C., Zaki, W., 2012. Non-linear dynamic thermomechanical behaviour of shape memory alloys. J. Intell. Mater. Syst. Struct. 23, 
$1593-1611$.

Nayfeh, A.H., Mook, D.T., 2008. Nonlinear Oscillations. John Wiley \& Sons.

Otsuka, K., Wayman, C.M., 1999. Shape Memory Materials. Cambridge University Press.

Piccirillo, V., Balthazar, J., Pontes Jr, B., 2010a. Analytical study of the nonlinear behavior of a shape memory oscillator: Part I-primary resonance and free response at low temperatures. Nonlinear Dyn. 59, 733-746.

Piccirillo, V., Balthazar, J., Pontes Jr, B., 2010b. Analytical study of the nonlinear behavior of a shape memory oscillator: Part II-resonance secondary. Nonlinear Dyn. 60, 513-524.

Piccirillo, V., Balthazar, J.M., Pontes, B.R., Felix, J.L.P., 2008. On a nonlinear and chaotic non-ideal vibrating system with shape memory alloy (SMA). Journal of Theoretical and Applied Mechanics 46, 597-620.

Piccirillo, V., Balthazar, J.M., Pontes, B.R., Felix, J.L.P., 2009a. Chaos control of a nonlinear oscillator with shape memory alloy using an optimal linear control: Part I: Ideal energy source. Nonlinear Dyn. 55, 139-149.

Piccirillo, V., Balthazar, J.M., Pontes, B.R., Felix, J.L.P., 2009b. Chaos control of a nonlinear oscillator with shape memory alloy using an optimal linear control: Part II: Nonideal energy source. Nonlinear Dyn. 56, 243-253.

Rao, C.N.R., Rao, K.J., 1978. Phase transitions in solids: an approach to the study of the chemistry and physics of solids. McGraw-Hill.

Saadat, S., Salichs, J., Noori, M., Hou, Z., Davoodi, H., Bar-On, I., Suzuki, Y., Masuda, A., 2002. An overview of vibration and seismic applications of NiTi shape memory alloy. Smart Mater. Struct. 11, 218.

Savi, M.A., 2015. Nonlinear dynamics and chaos in shape memory alloy systems. Int. J. Non-linear Mech. 70, 2-19.

Savi, M.A., Nogueira, J.B., 2010. Nonlinear dynamics and chaos in a pseudoelastic two-bar truss. Smart Mater. Struct. 19, 115022.

Savi, M.A., Pacheco, P.M.C.L., 2002. Chaos and hyperchaos in shape memory systems. Int. J. Bifurcation Chaos 12, 645-657.

Savi, M.A., Pacheco, P.M.C.L., Braga, A.M.B., 2002. Chaos in a shape memory two-bar truss. Int. J. Non-linear Mech. 37, 1387-1395.

Scheffer, M., Carpenter, S., Foley, J.A., Folke, C., Walker, B., 2001. Catastrophic shifts in ecosystems. Nature 413, 591-596.

Scheffer, M., Carpenter, S.R., 2003. Catastrophic regime shifts in ecosystems: linking theory to observation. Trends in Ecology and Evolution 18, 648-656.

Schmidt, G., Tondl., A., 1986. Non-linear Vibrations. Cambridge University Press.

Schmidt, I., Lammering, R., 2004a. The damping behaviour of superelastic NiTi components. Mat Sci Eng a-Struct 378, 70-75.

Schmidt, I., Lammering, R., 2004b. Experimental investigations on the damping behaviour of superelastic NiTi. J. Phys. IV 115, 11-20. 
Seelecke, S., 2002. Modeling the dynamic behavior of shape memory alloys. Int. J. Non-linear Mech. 37, 1363-1374.

Shaw, J.A., Kyriakides, S., 1995. Thermomechanical Aspects of Niti. J. Mech. Phys. Solids 43, 1243-1281.

Stoker, J.J., 1966. Nonlinear Vibrations.

Timoshenko, S., Goodier, J.N., 1969. Theory of elasticity. McGraw-Hill.

Van Humbeeck, J., 2003. Damping capacity of thermoelastic martensite in shape memory alloys. J. Alloys Compd. 355, 58-64.

Xia, M., Sun, Q., 2015. Jump phenomena of rotational angle and temperature of NiTi wire in nonlinear torsional vibration. Int. J. Solids Struct. 56-57, 220-234.

Yin, H., He, Y.J., Sun, Q.P., 2014. Effect of deformation frequency on temperature and stress oscillations in cyclic phase transition of NiTi shape memory alloy. J. Mech. Phys. Solids 67, 100-128. 
Table 1. Mechanical and thermal properties of the material.

\begin{tabular}{c|c|c}
\hline Austenite finish temperature & $A_{f}$ & $18.51^{\circ} \mathrm{C}$ \\
\hline Shear modulus in austenite phase & $G$ & $16.5 \mathrm{GPa}$ \\
\hline $\begin{array}{c}\text { Shear strain at the start of } \\
\text { phase transition }\end{array}$ & $\gamma_{s}$ & $1.10 \%$ \\
Maximum phase transition & $\gamma_{0}^{P T}$ & $10.00 \%$ \\
\hline shear strain & $k$ & $18.3 \mathrm{~W} / \mathrm{K} \cdot \mathrm{m}$ \\
\hline Specific latent heat & $l_{0}$ & $7.74 \times 10^{7} \mathrm{~J} / \mathrm{m}^{3}$ \\
\hline Specific heat capacity & $\lambda=\rho c_{p}$ & $3.225 \times 10^{6} \mathrm{~J} / \mathrm{m}^{3} \cdot \mathrm{K}$ \\
\hline
\end{tabular}




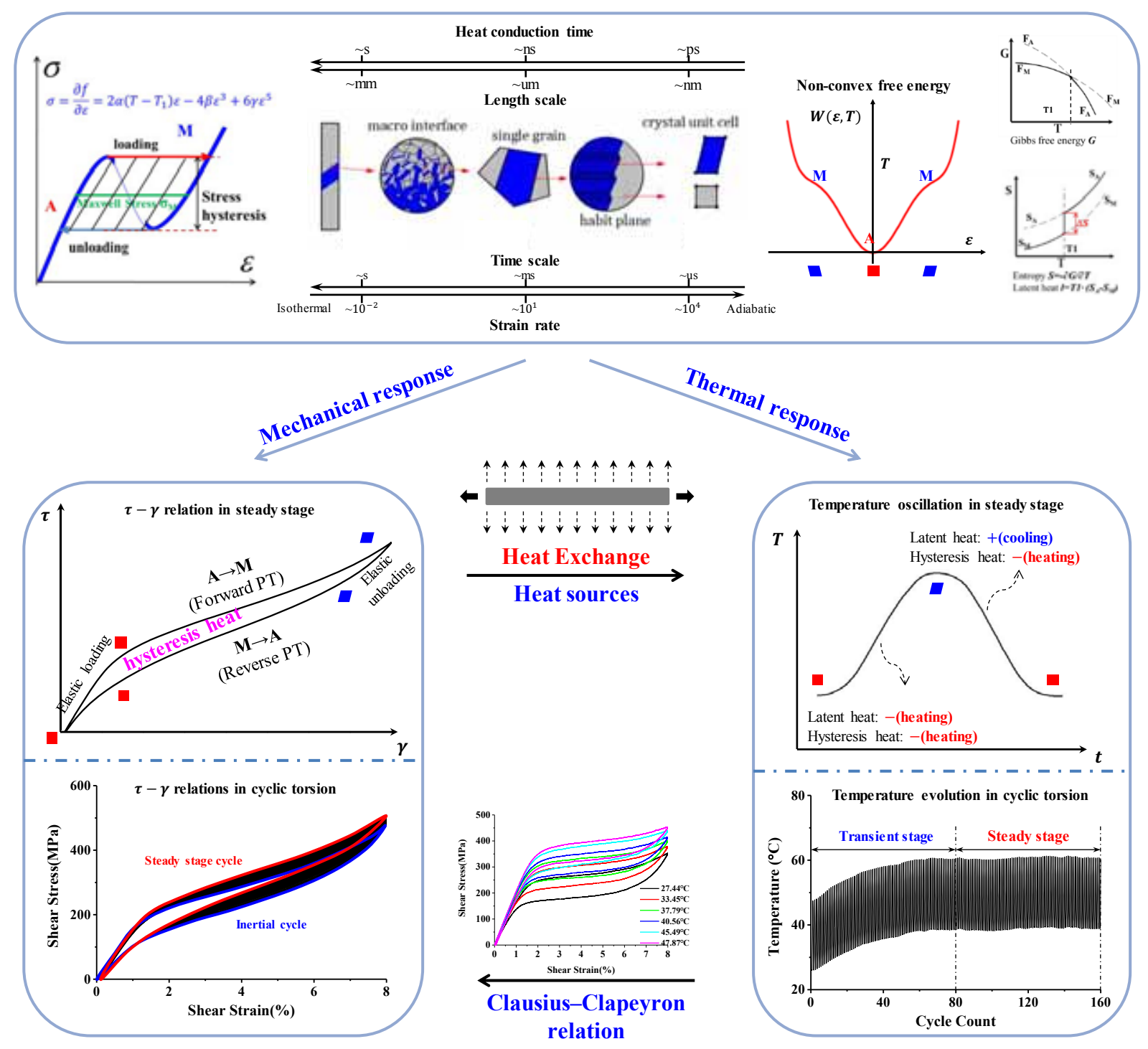

Figure 1. Physics of phase transition and thermomechanical coupling effects in superelastic NiTi SMA. 
(a) Dynamic response of a softening/linear/hardening vibration system in the frequency and amplitude domain
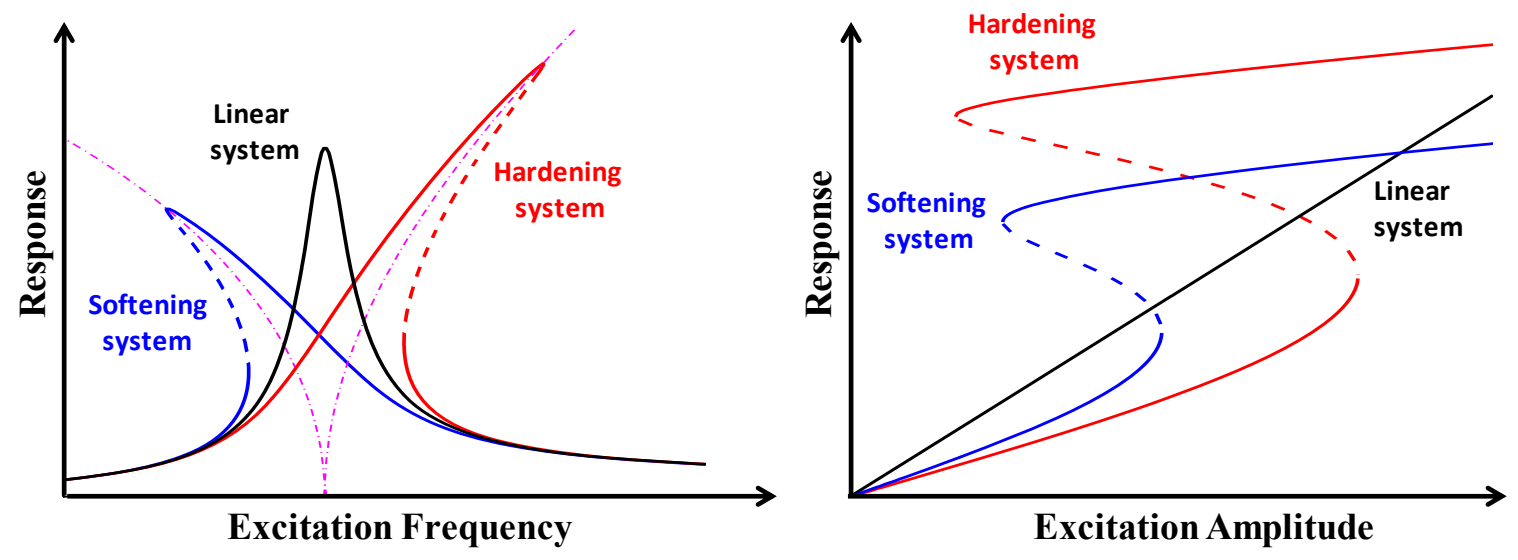

(b) Jump phenomenon of a softening nonlinear vibration system

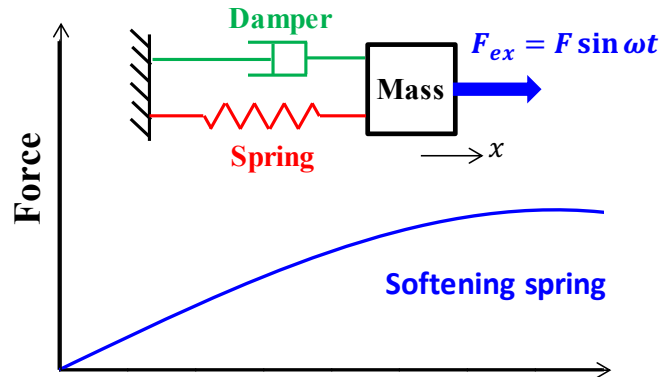

\section{Displacement}
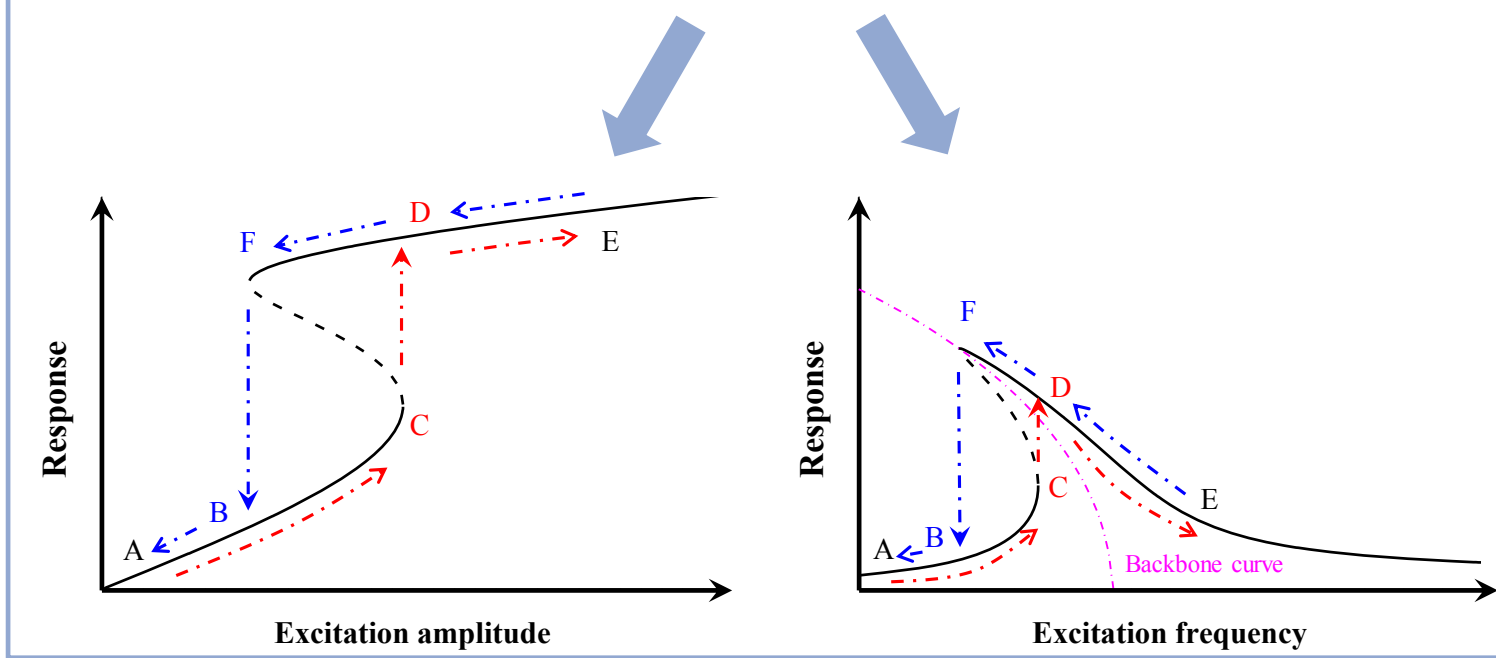

Figure 2. (a) FRC and ARC of a softening/linear/hardening vibration system; (b) jump phenomenon of a softening nonlinear vibration system in the frequency and amplitude domains. 

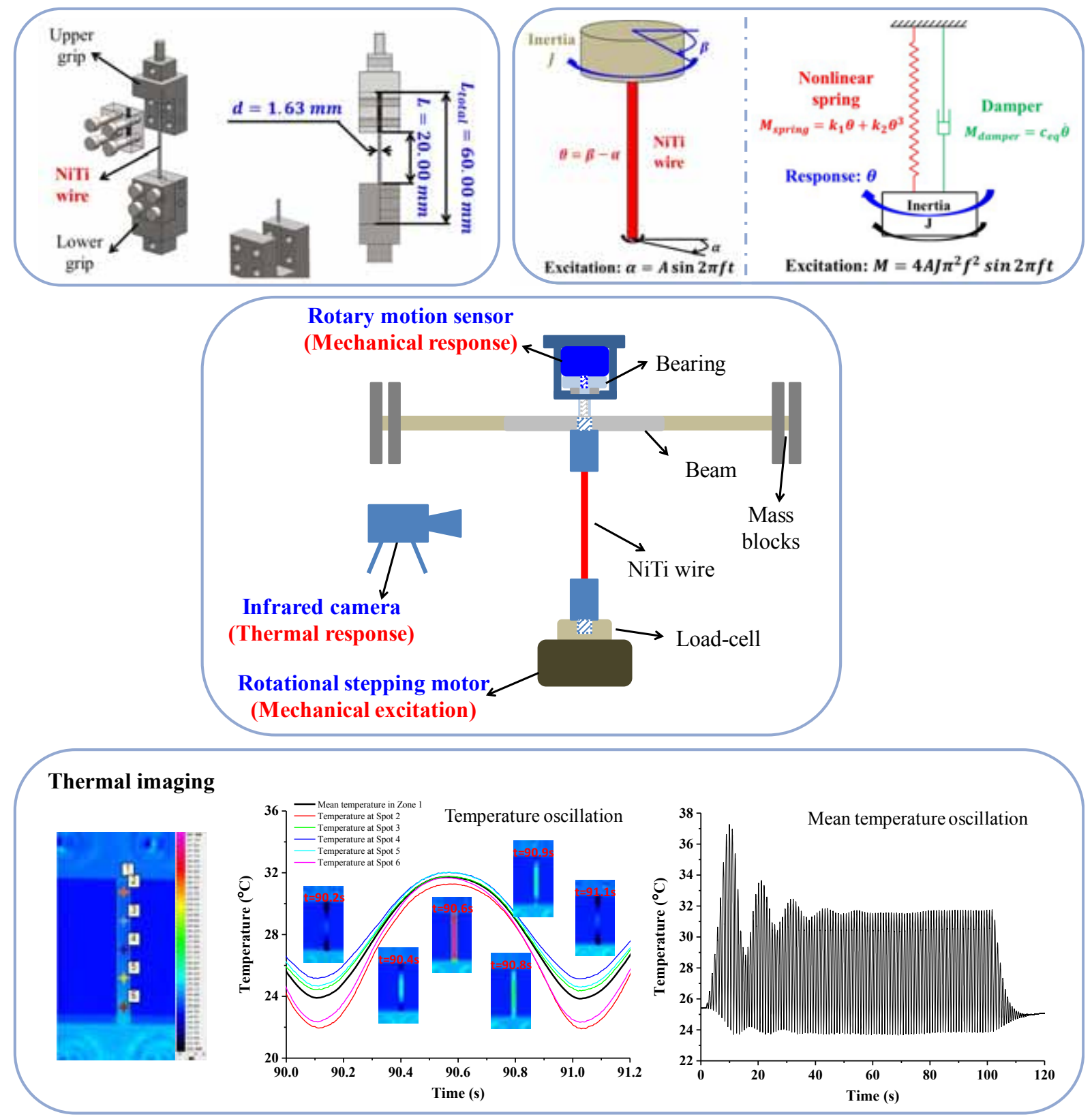

Figure 3. Experimental setup and synchronized measurement of thermomechanical responses in the nonlinear torsional vibration system. 
(a)
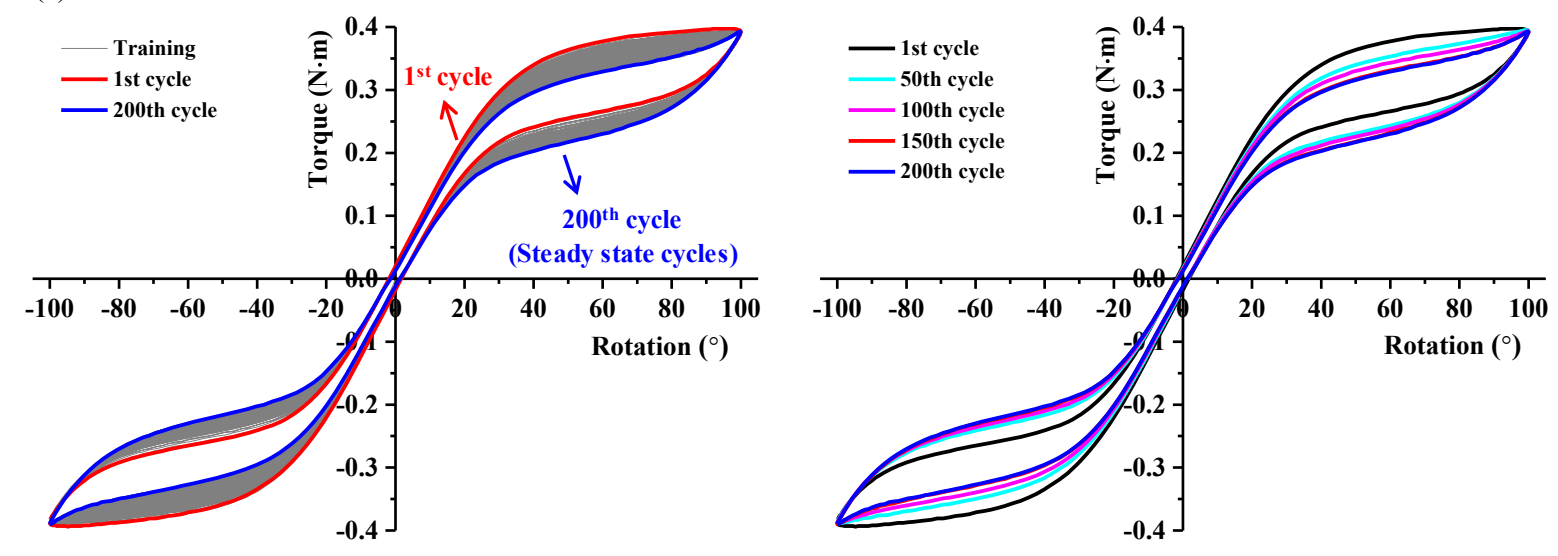

(b)
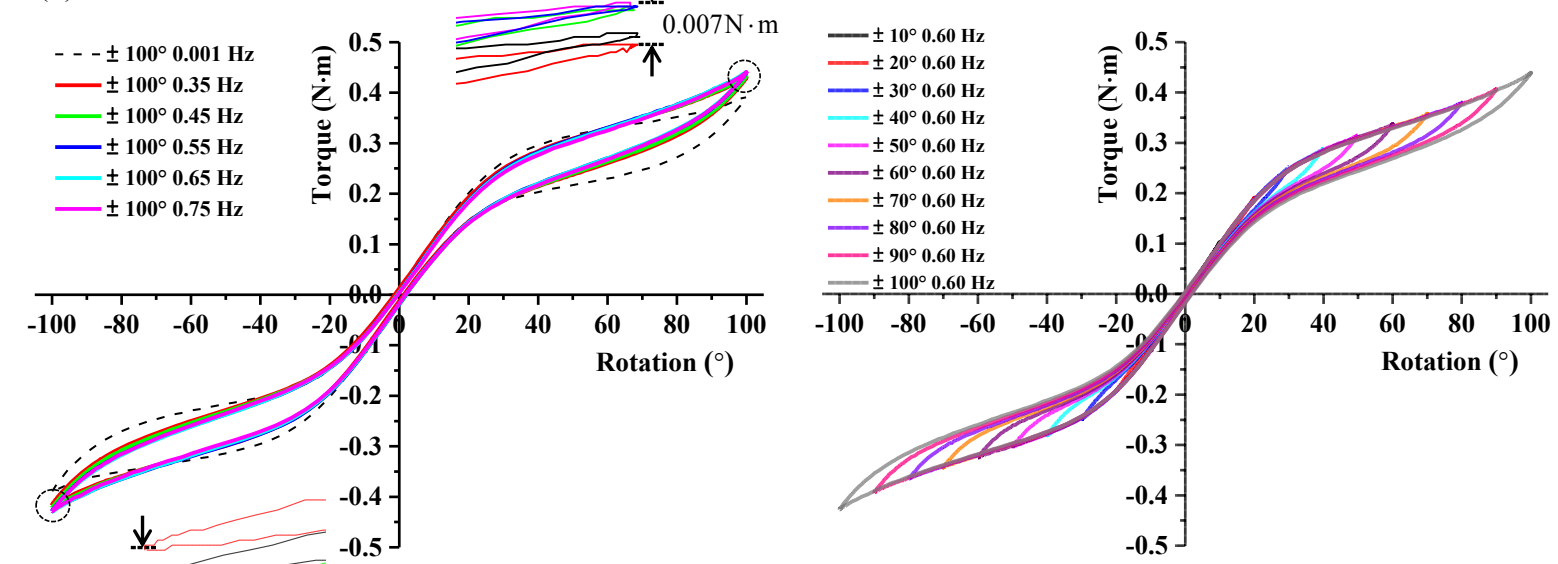

$0.011 \mathrm{~N} \cdot \mathrm{m}$

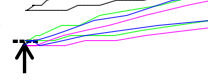

(c)

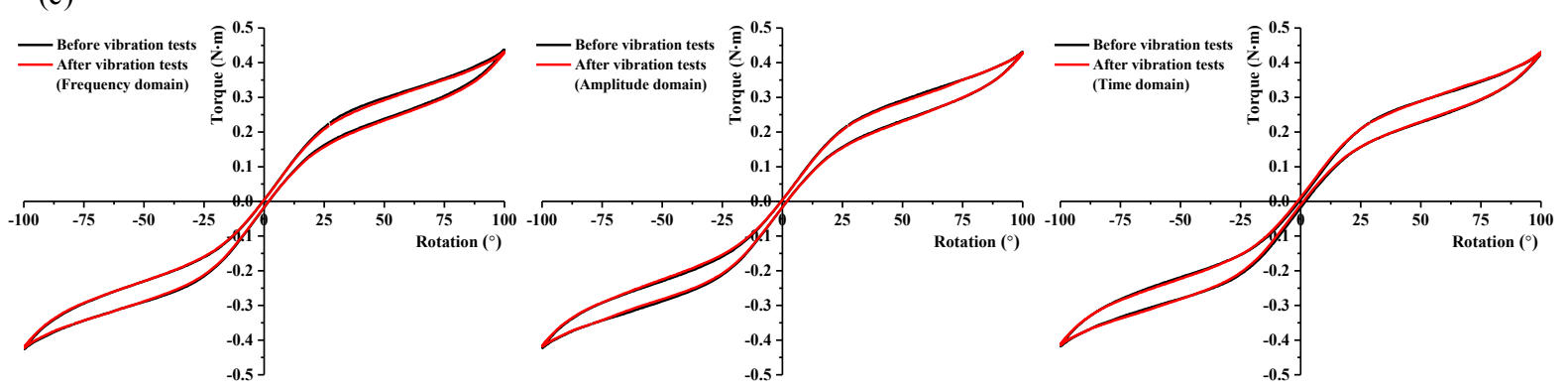

Figure 4. (a) Pre-test training of the specimen under $\varphi= \pm 100^{\circ}$ and $f=0.001 \mathrm{~Hz}$; (b) characterization of the torque-angle relations in pure torsion at different frequencies and rotations; (c) comparison of the steady state torque-angle relations of the three specimens before and after the torsional vibration tests in the frequency, amplitude and time domains. 


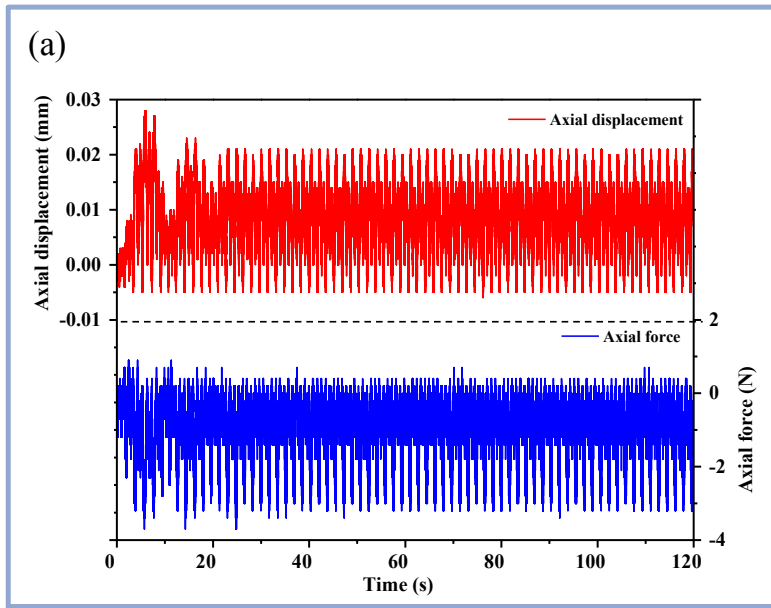

(b)
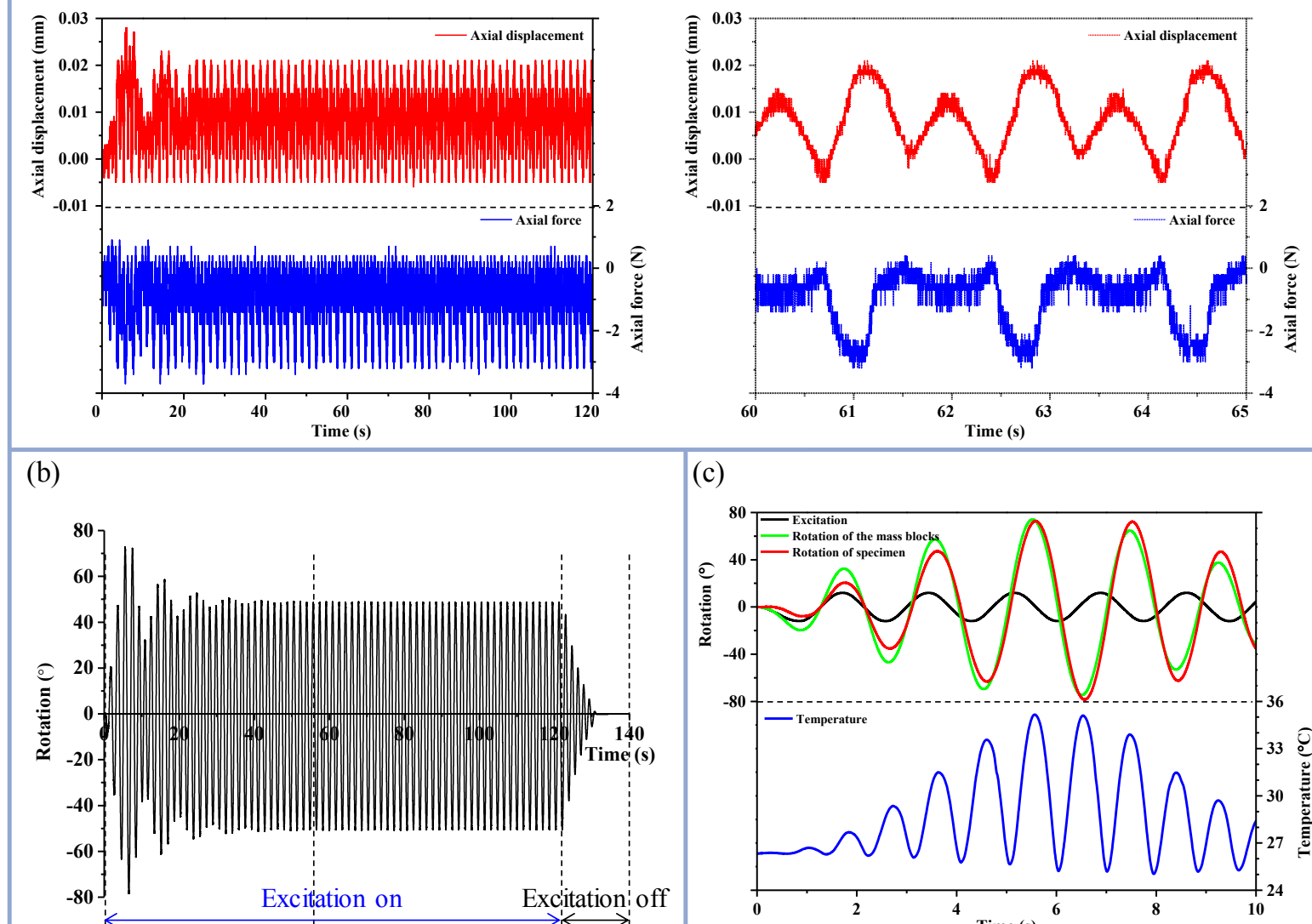

(c)
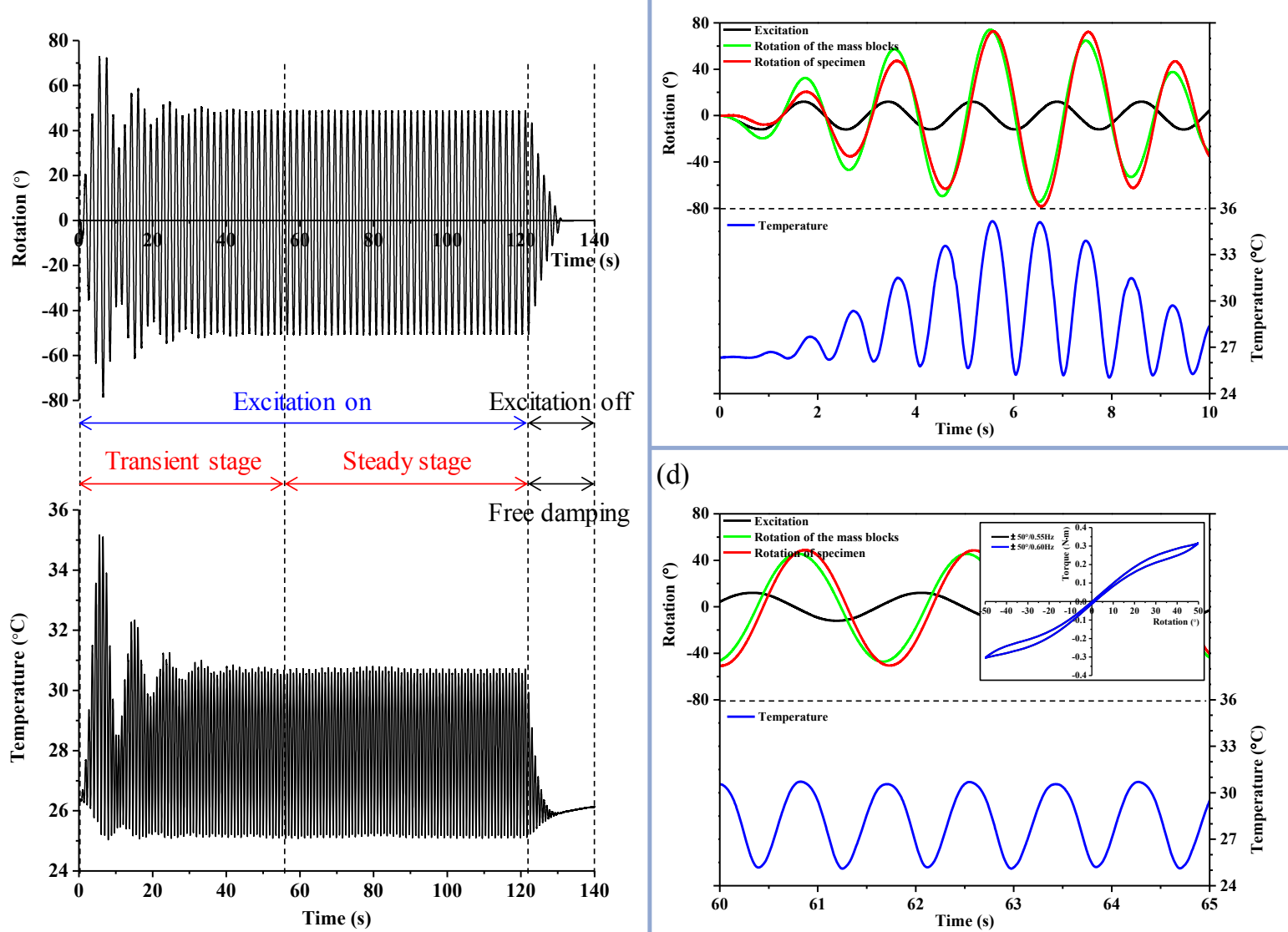

(d)

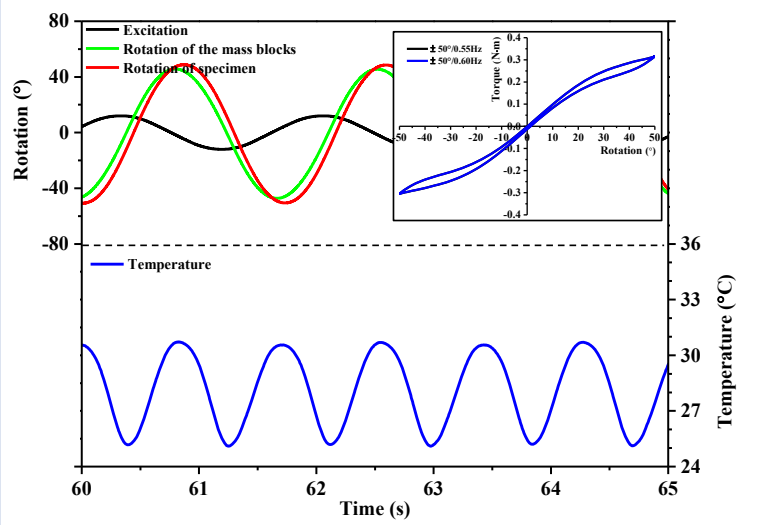

Figure 5. (a) Axial force and axial displacement of the NiTi wire during torsional vibration test; (b) evolutions of $\theta(t)$ and $T(t)$ in the time domain; (c) synchronization of $\alpha(t)$, $\beta(t), \theta(t)$ and $T(t)$ in the transient state and (d) oscillation of $\theta(t)$ and $T(t)$ in the steady state. 
(a) Experiments and modeling of the FRCs of rotation
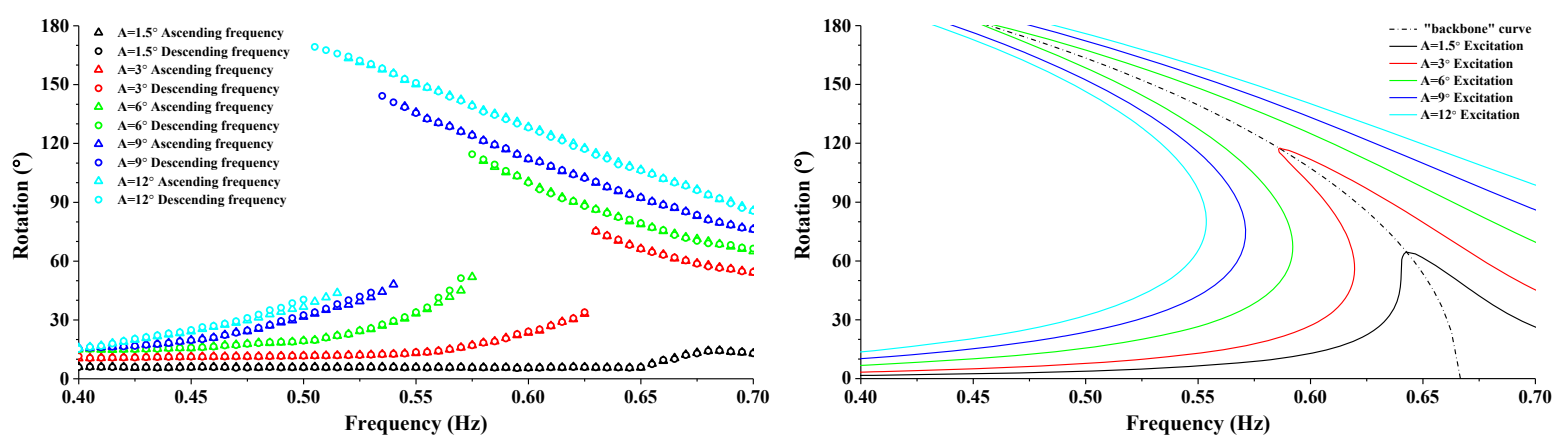

(b) Experiments and modeling of the FRCs of temperature
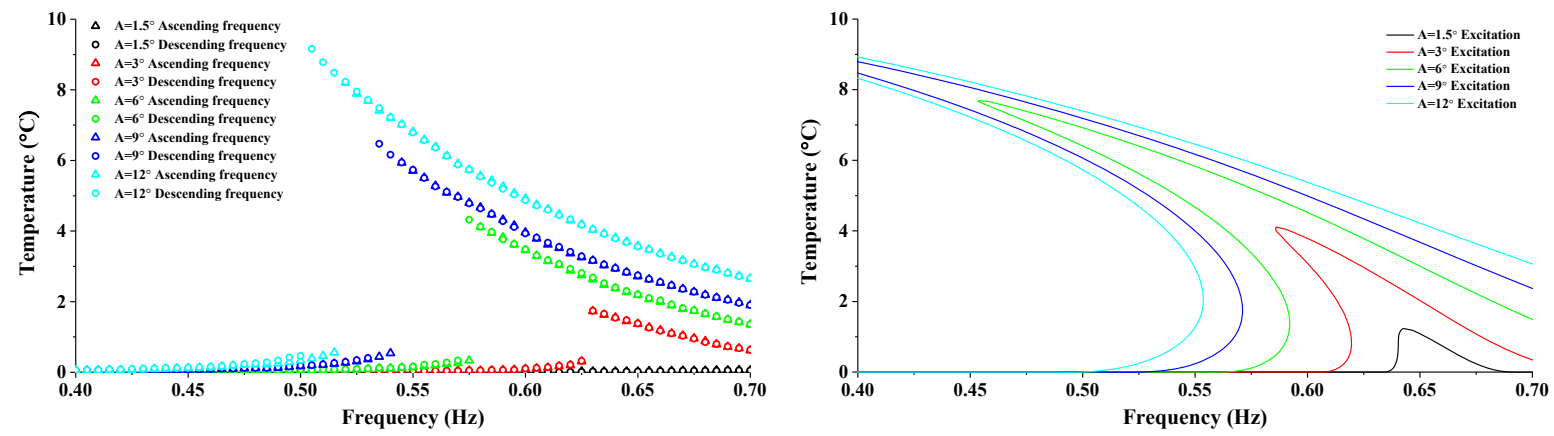

Figure 6. (a) Comparison of the steady state $\Delta \theta$ between the experimental data and theoretical modeling under the excitation of $A=1.5^{\circ} / 3^{\circ} / 6^{\circ} / 9^{\circ} / 12^{\circ}$; (b) comparison of the steady state $\Delta T$ between the experimental data and theoretical modeling under the excitation of $A=1.5^{\circ} / 3^{\circ} / 6^{\circ} / 9^{\circ} / 12^{\circ}$. 
(a) Experimental results
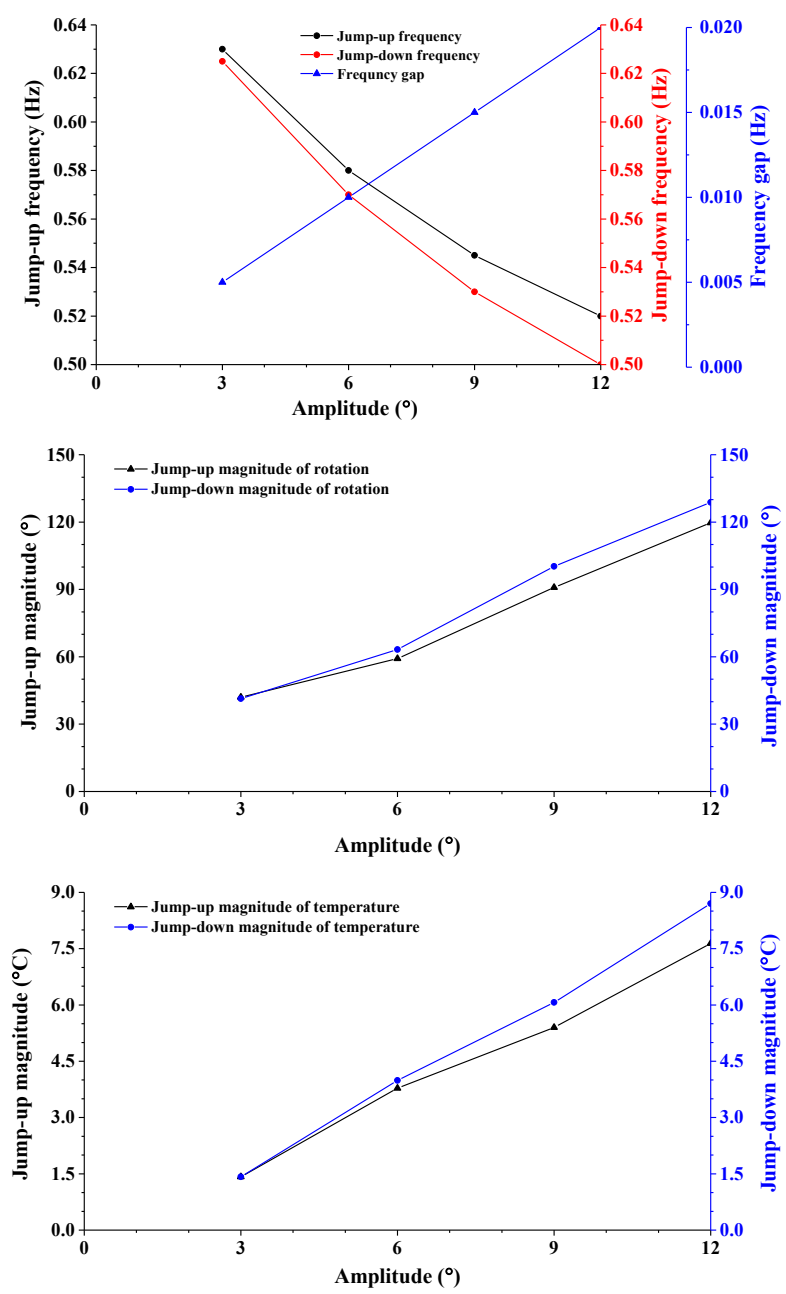

(b) Modeling results
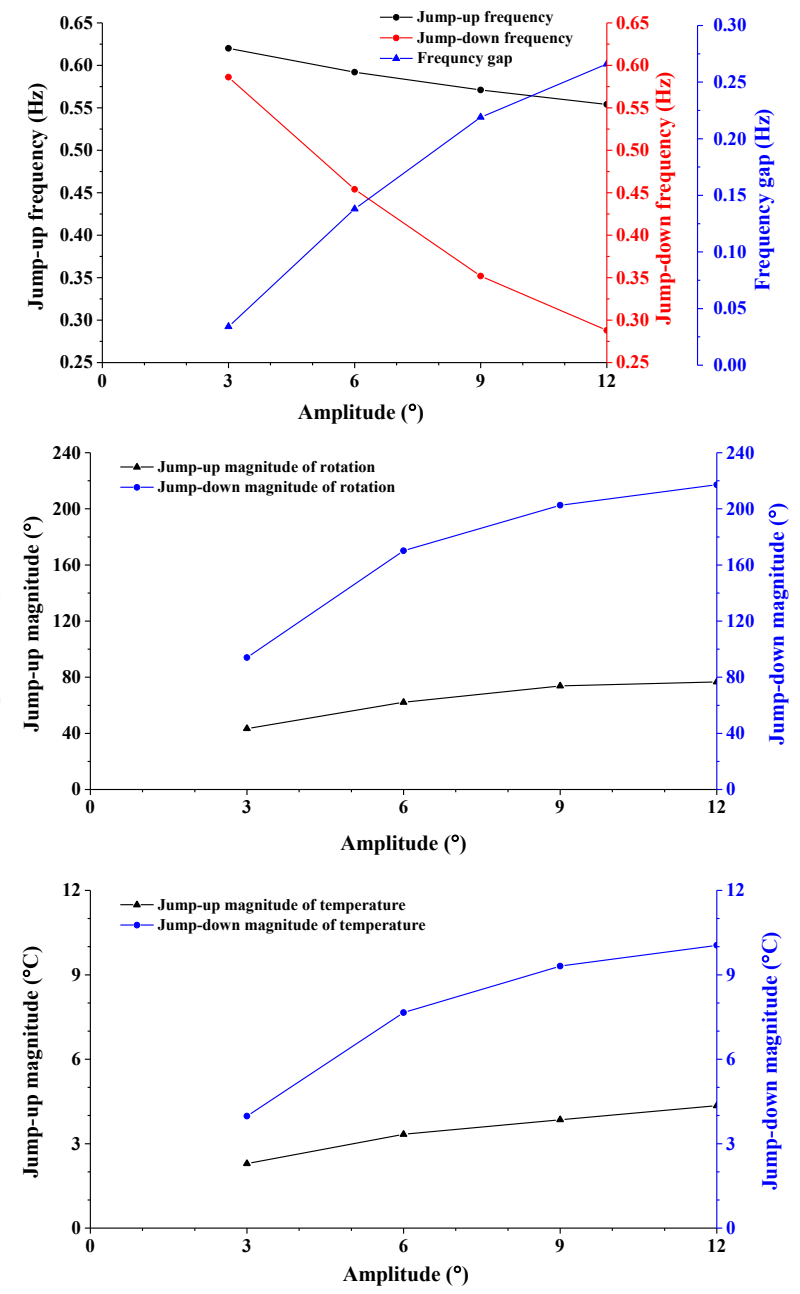

Figure 7. (a) Experimental data and (b) theoretical modeling results of the jump frequencies $\left(f_{\text {up }}\right.$ and $\left.f_{\text {down }}\right)$, jump frequency gap $(\Delta f)$, jump magnitude of rotational angle $\left(\Delta \theta_{u p}\right.$ and $\left.\Delta \theta_{\text {down }}\right)$ and jump magnitude of temperature $\left(\Delta T_{\text {up }}\right.$ and $\left.\Delta T_{\text {down }}\right)$ at different excitation amplitudes. 
(a) Effective Duffing oscillation model

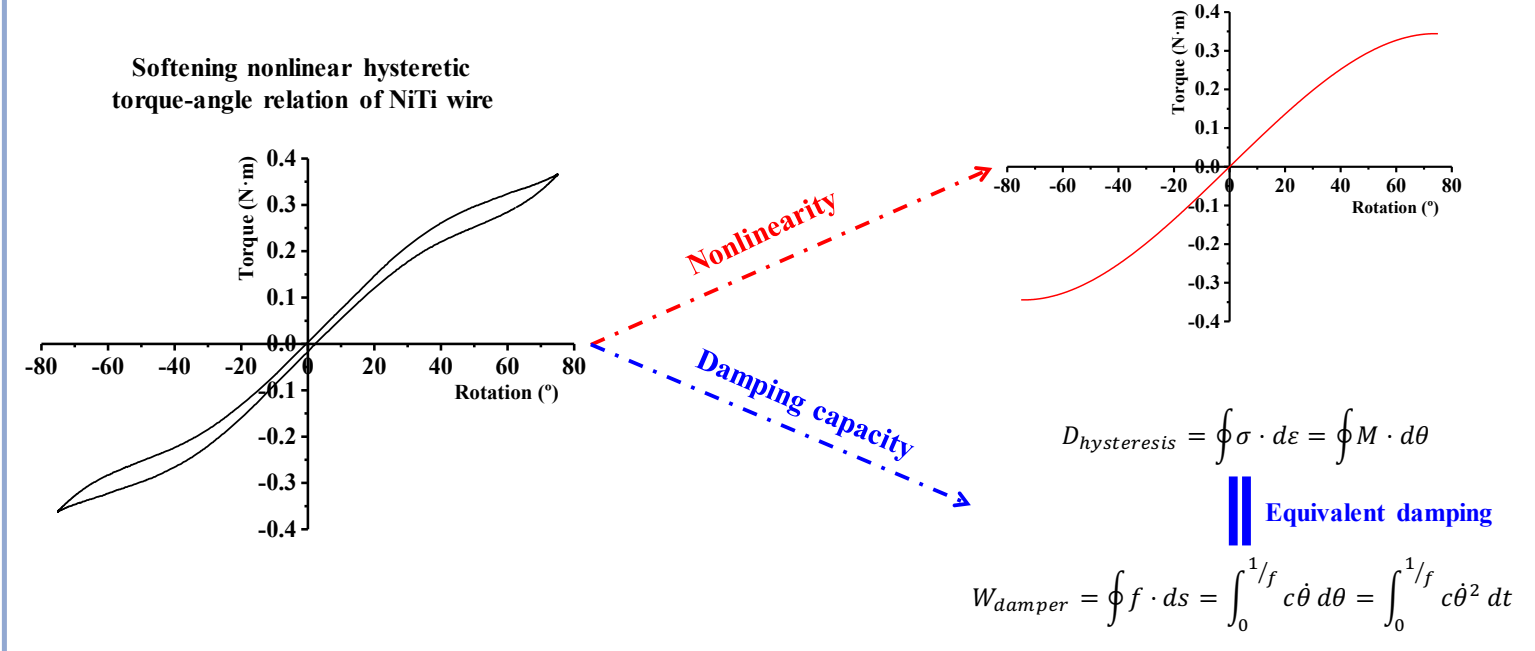

(b) Ideal "elastoplastic" deformation with perfect plasticity in torsion

Linear elastic deformation

(small rotation)

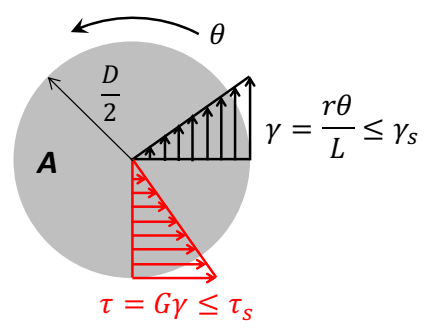

"Elastoplastic" deformation (large rotation)

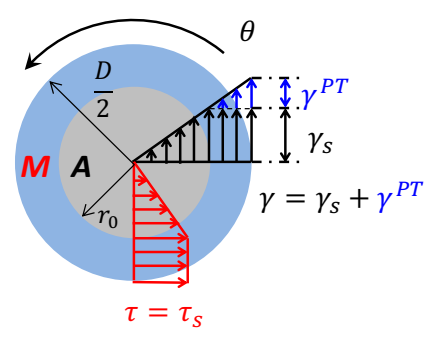

"Elastoplastic" $\tau-\gamma$ relation with perfect plasticity

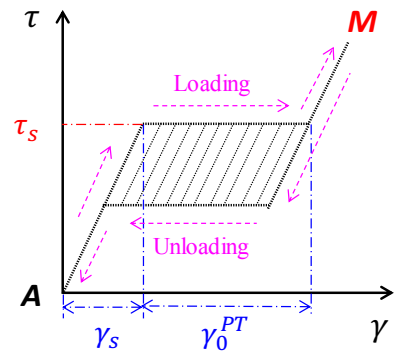

Figure 8. (a) Simplification of the softening nonlinear hysteretic behavior of NiTi wire to an effective Duffing oscillator and an equivalent viscous damper; (b) determination of heat source based on the ideal "elastoplastic" deformation with perfect plasticity of NiTi wire in partial phase transition during torsion. 
(a) Experiments and modeling of the ARCs of rotation
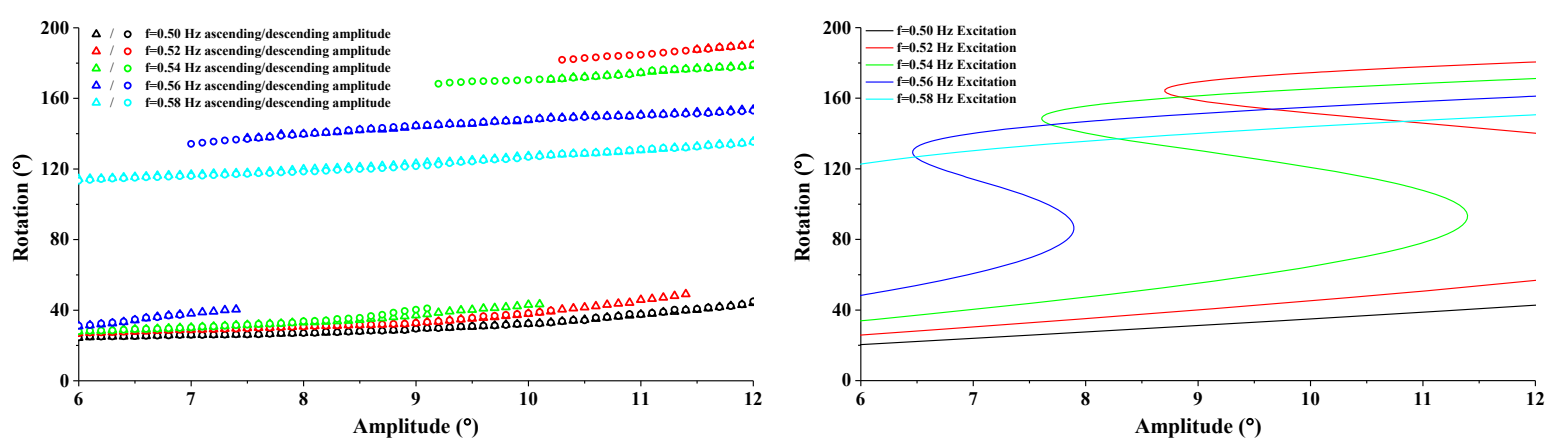

(b) Experiments and modeling of the ARCs of temperature
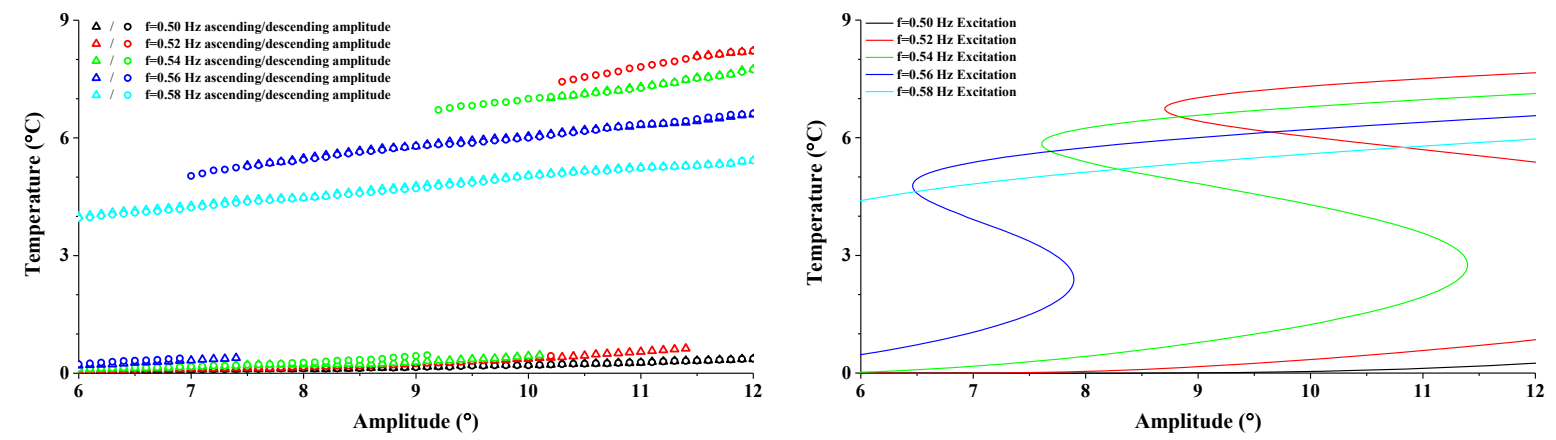

Figure 9. (a) Comparison of the steady state $\Delta \theta$ between the experimental data and theoretical modeling under the excitation of $f=0.50 \mathrm{~Hz} / 0.52 \mathrm{~Hz} / 0.54 \mathrm{~Hz} / 0.56 \mathrm{~Hz} / 0.58 \mathrm{~Hz}$; (b) comparison of the steady state $\Delta T$ between the experimental data and theoretical modeling under the excitation of $f=0.50 \mathrm{~Hz} / 0.52 \mathrm{~Hz} / 0.54 \mathrm{~Hz} / 0.56 \mathrm{~Hz} / 0.58 \mathrm{~Hz}$. 
(a) Experimental results
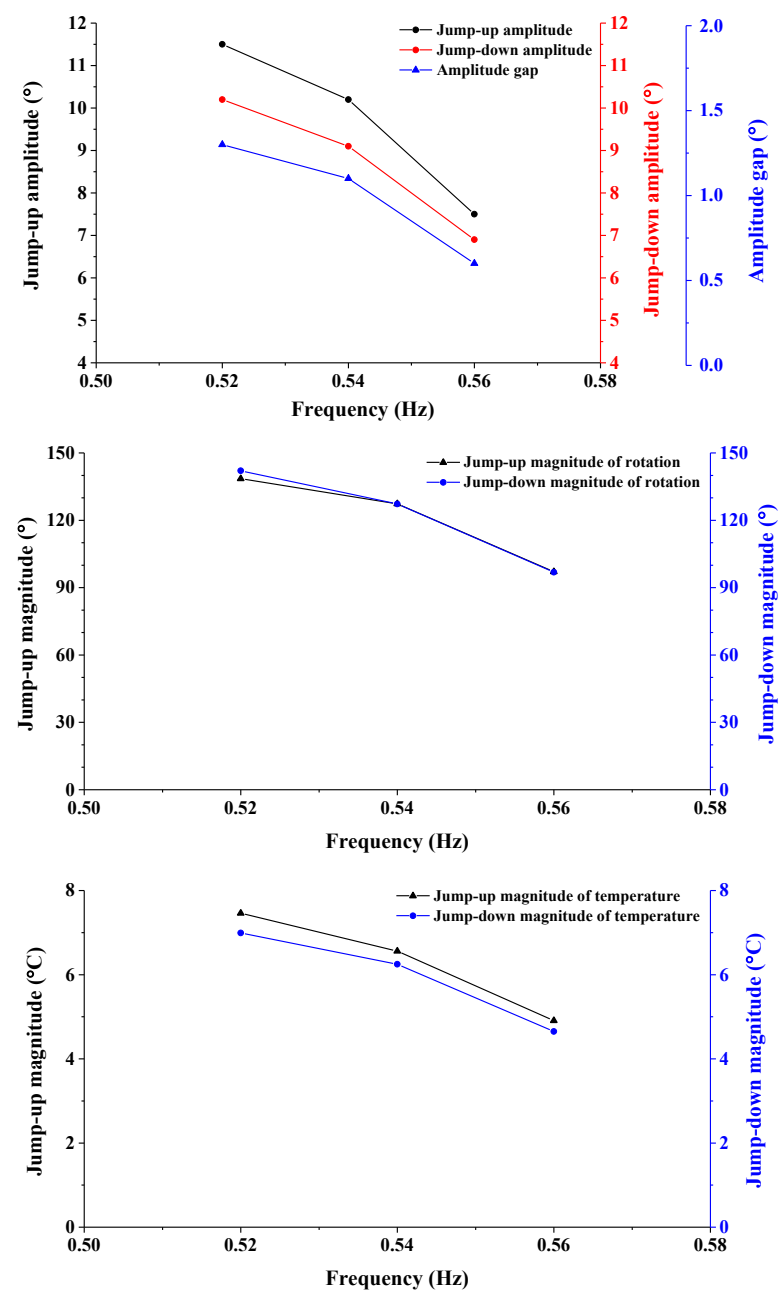

(b) Modeling results
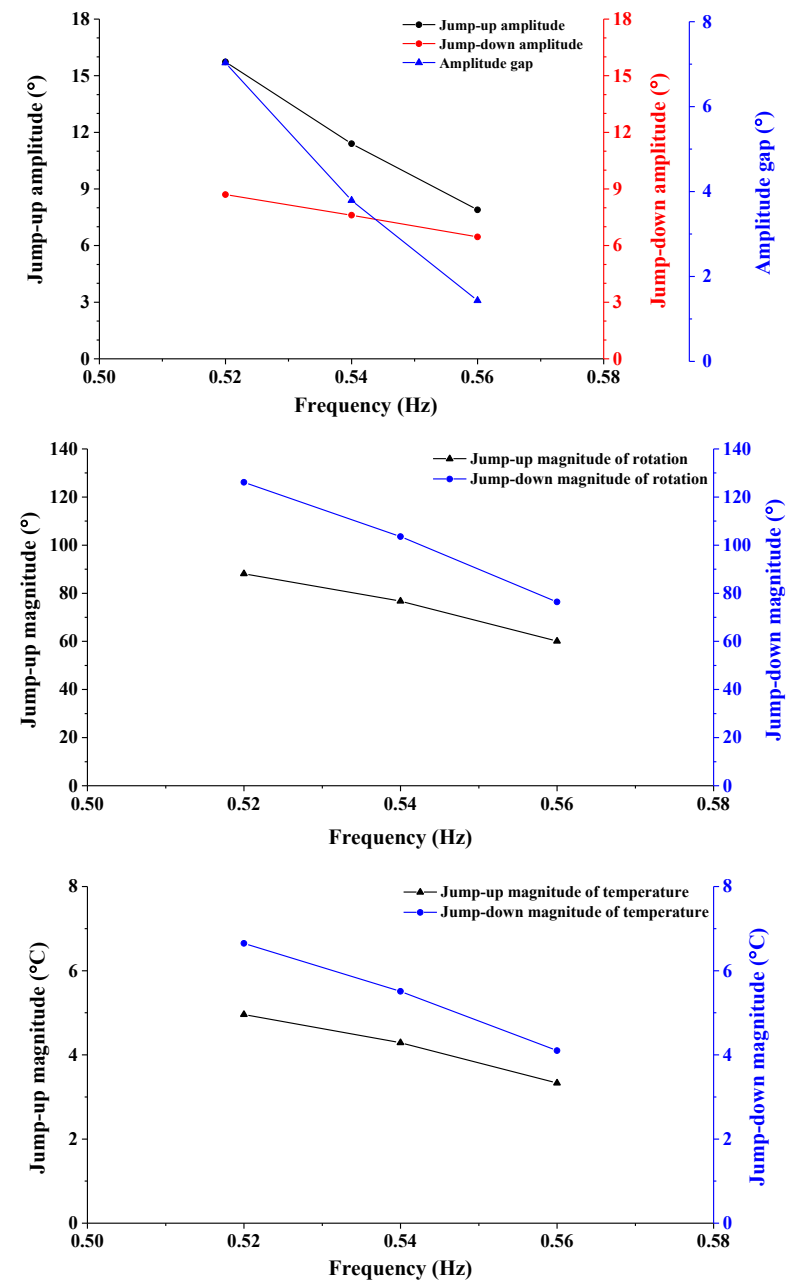

Figure 10. (a) Experimental data and (b) theoretical modeling results of the jump amplitudes $\left(A_{\text {up }}\right.$ and $A_{\text {down }}$ ), jump amplitude gap ( $\left.\Delta A\right)$, jump magnitude of rotational angle $\left(\Delta \theta_{u p}\right.$ and $\left.\Delta \theta_{\text {down }}\right)$ and jump magnitude of temperature $\left(\Delta T_{\text {up }}\right.$ and $\left.\Delta T_{\text {down }}\right)$ at different excitation frequencies. 
(a) $A=3^{\circ}$ and $f=0.62160 \mathrm{~Hz}$

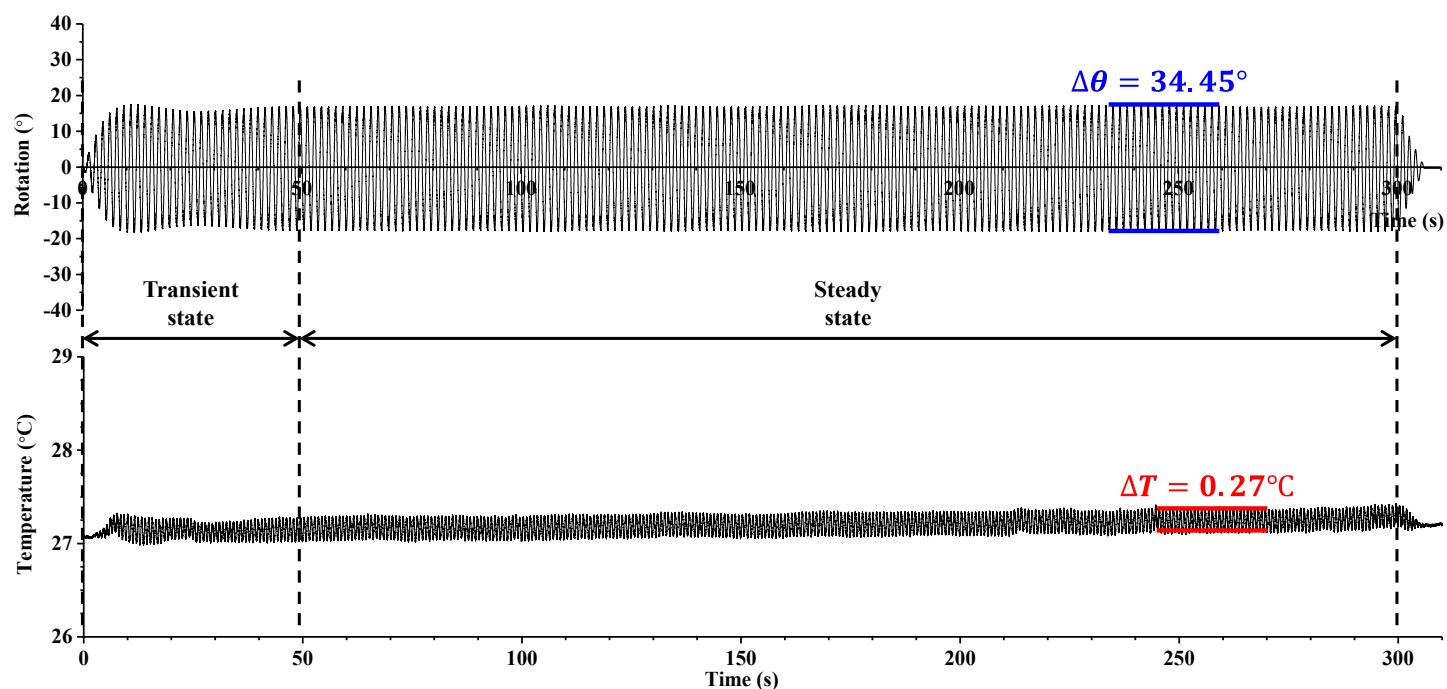

(b) $A=3^{\circ}$ and $f=0.62180 \mathrm{~Hz}$

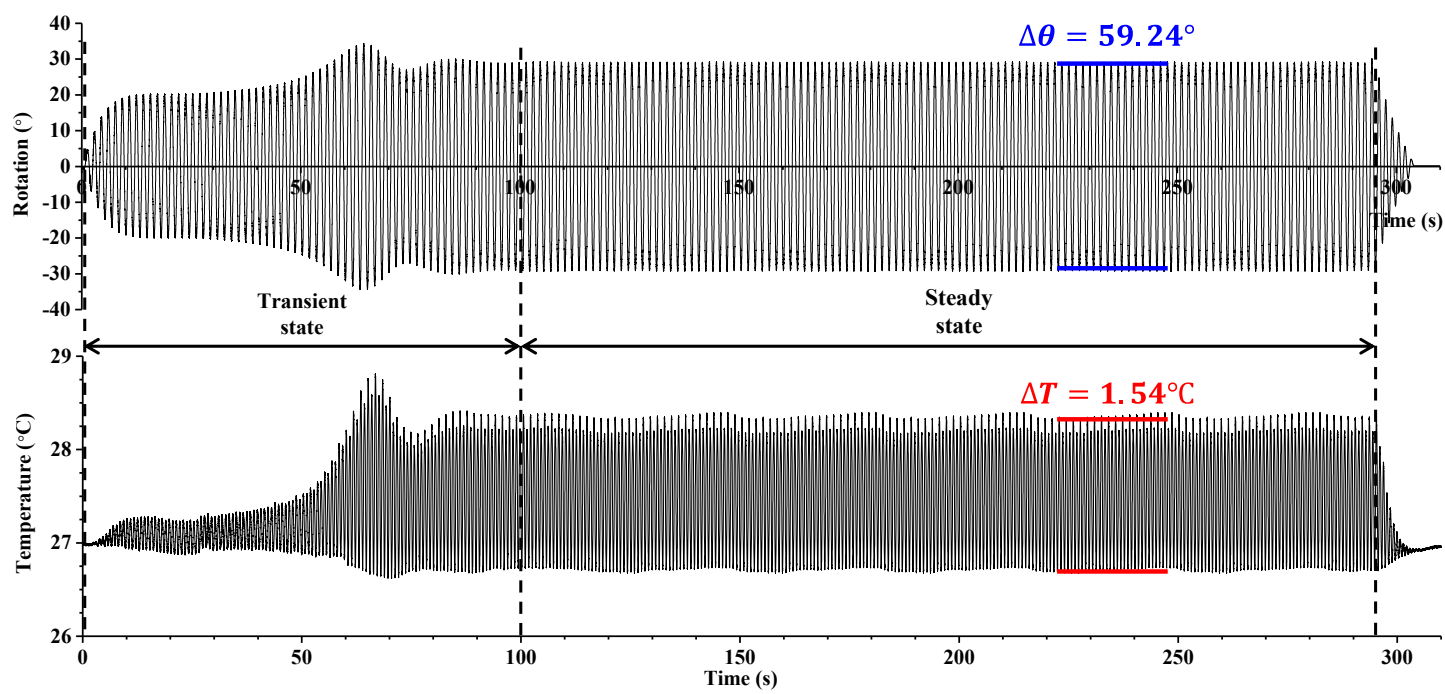

(c) $A=3^{\circ}$ and $f=0.62170 \mathrm{~Hz}$

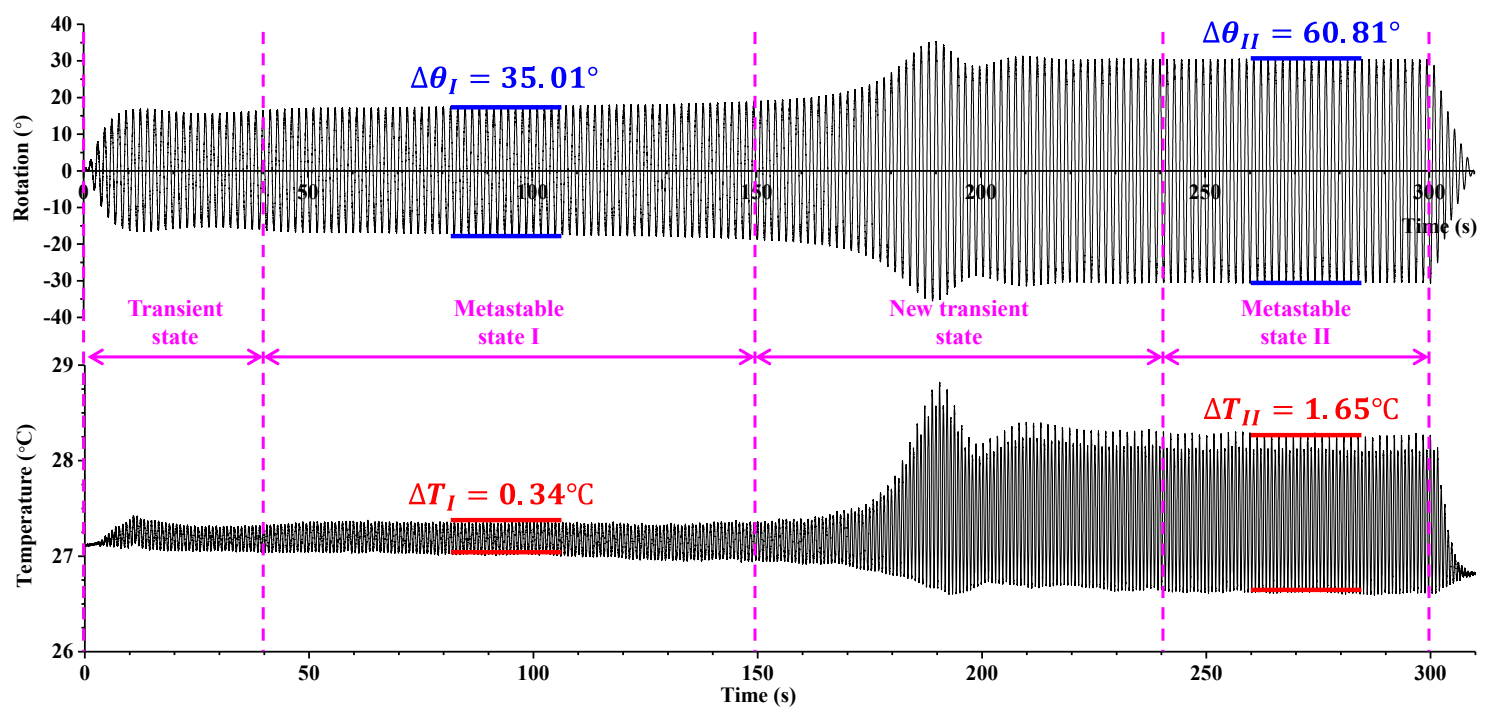

Figure 11. Evolutions of $\theta(t)$ and $T(t)$ of the dynamic system under the excitation of $A=3^{\circ}$ and (a) $f=0.62160 \mathrm{~Hz}$, (b) $f=0.62180 \mathrm{~Hz}$, (c) $f=0.62170 \mathrm{~Hz}$. 


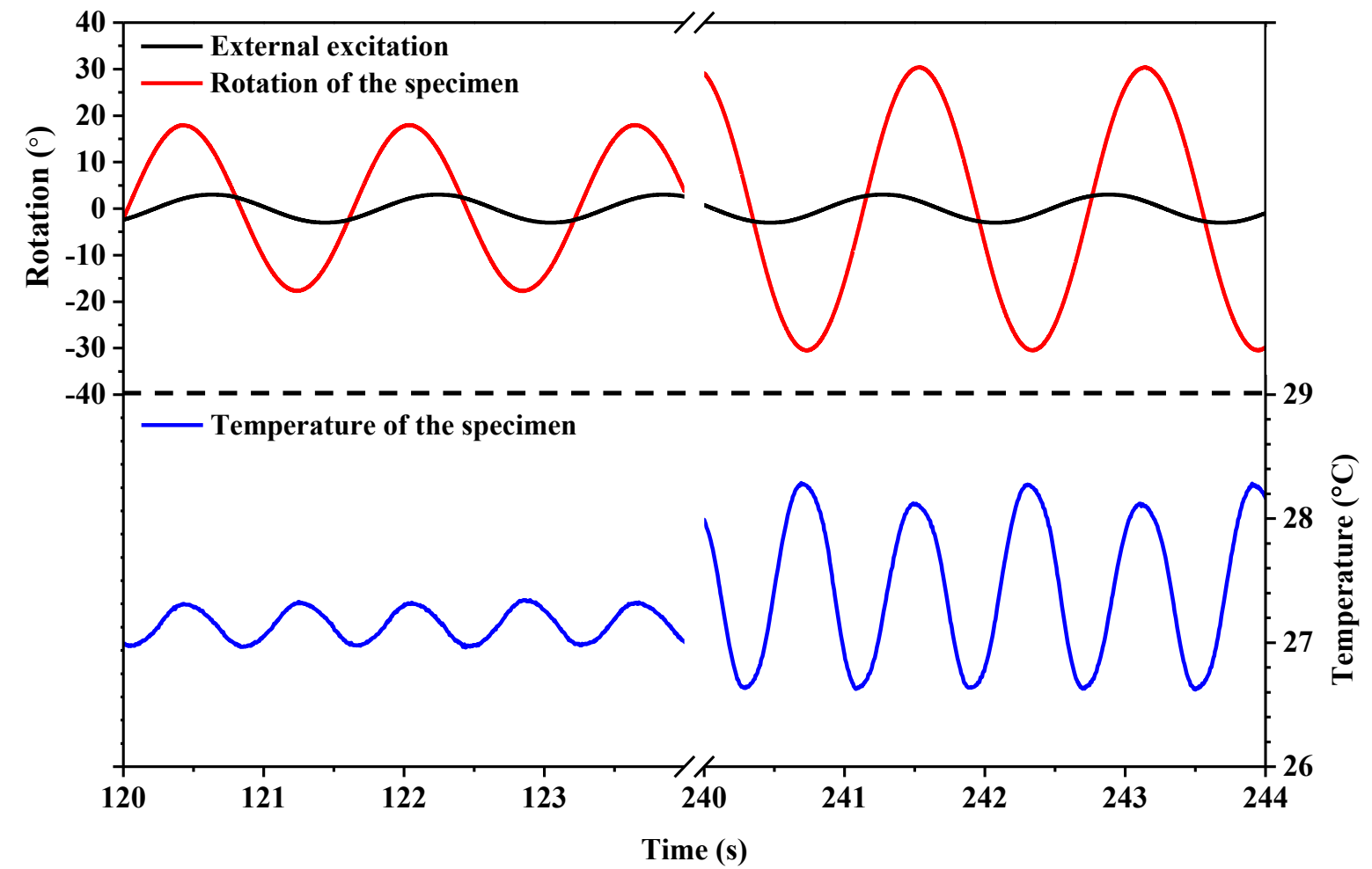

Figure 12. Comparison of the oscillations of $\theta(t)$ and $T(t)$ between metastable state I and metastable state II. 
(a) $A=9.0^{\circ}$ and $f=0.450 \mathrm{~Hz}$ (stable lower branch in the frequency domain)
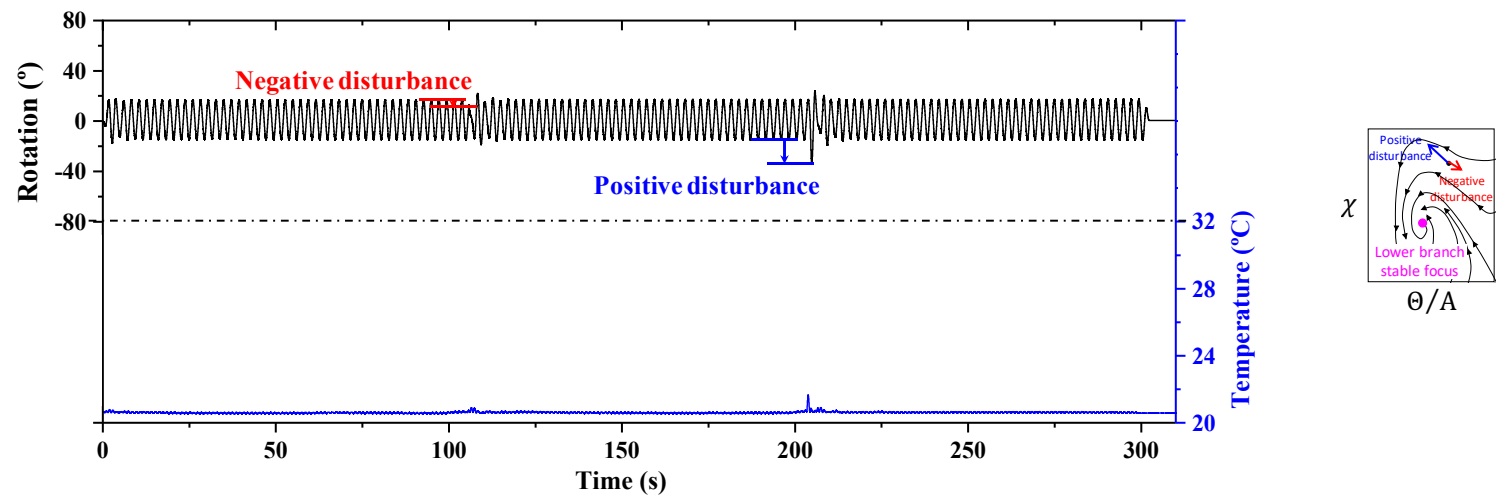

(b) $A=9.0^{\circ}$ and $f=0.600 \mathrm{~Hz}$ (stable upper branch in the frequency domain)
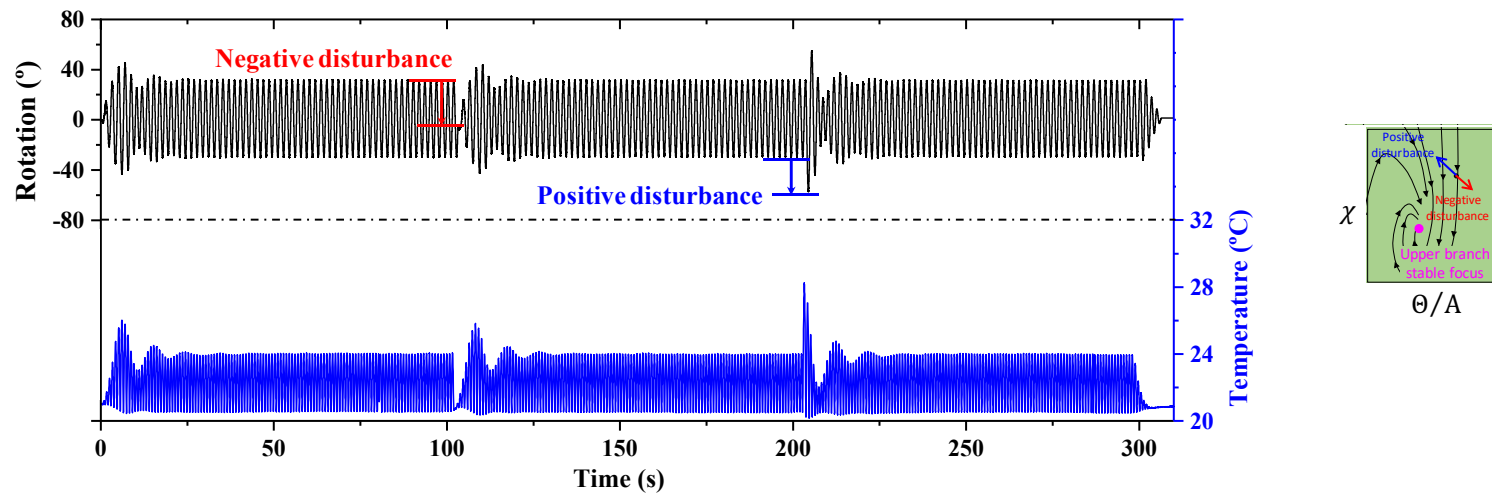

$\Theta / \mathrm{A}$

(c) $A=9.0^{\circ}$ and $f=0.531 \mathrm{~Hz}$ (metastable region in the frequency domain)
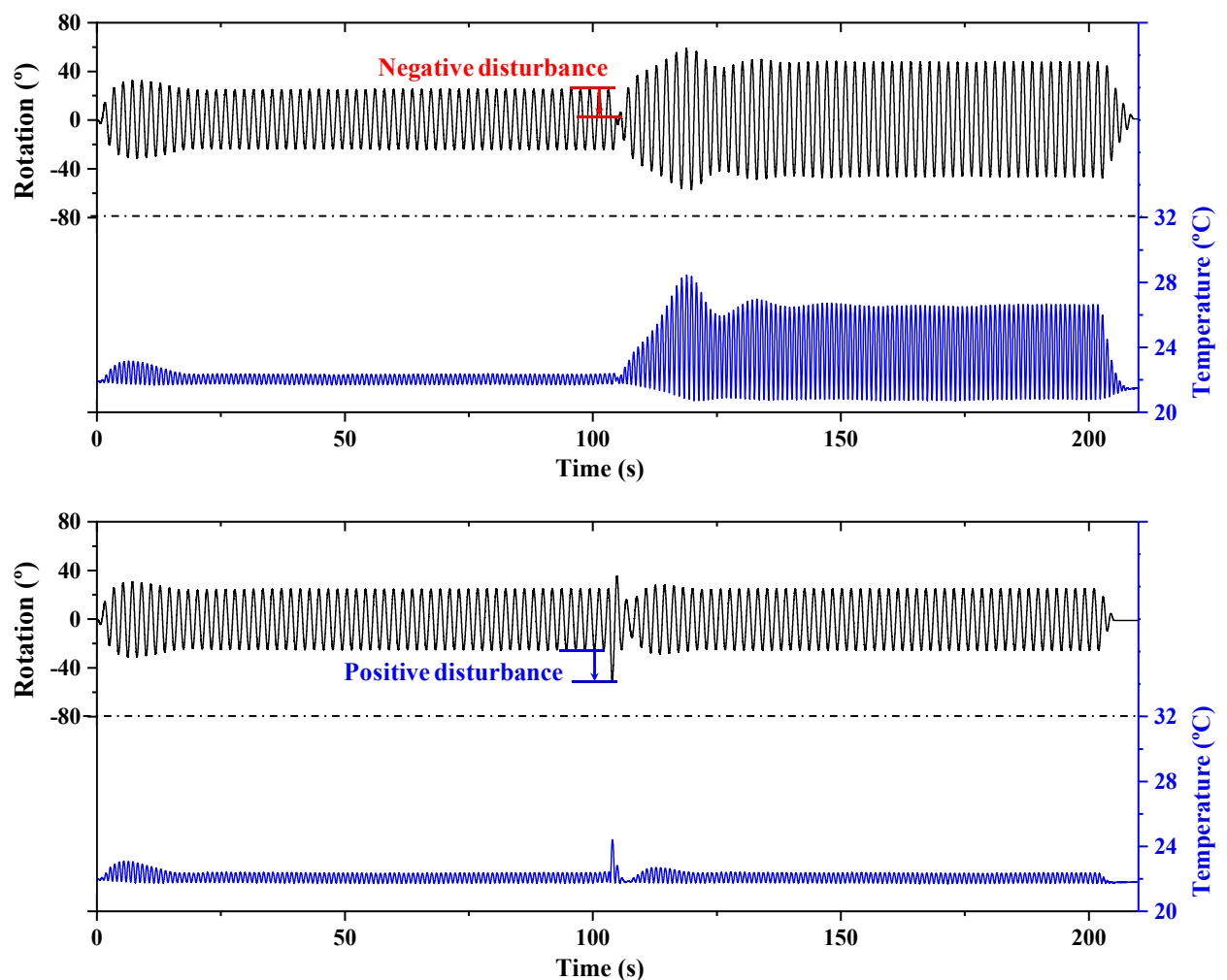

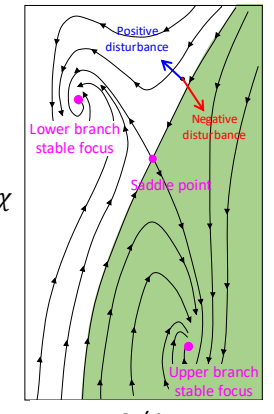

$\Theta / \mathrm{A}$

Figure 13. Evolutions of $\theta(t)$ and $T(t)$ at the excitation of $A=9.0^{\circ}$ and (a) $f=0.450 \mathrm{~Hz}$, (b) $f=0.600 \mathrm{~Hz}$, (c) $f=0.531 \mathrm{~Hz}$ with external negative and positive disturbances. 
(a) $A=6.0^{\circ}$ and $f=0.56 \mathrm{~Hz}$ (stable lower branch in the amplitude domain)

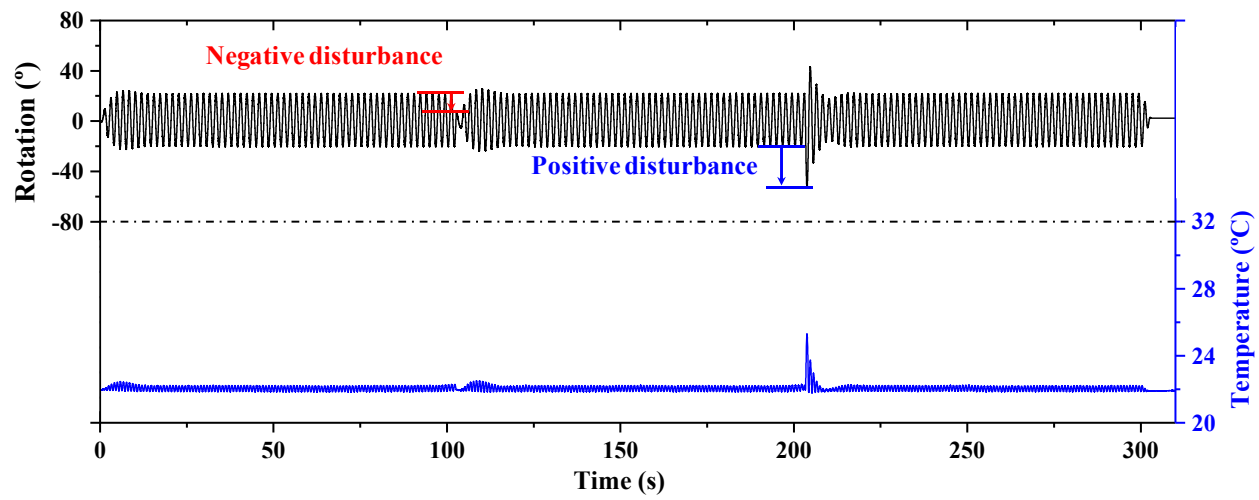

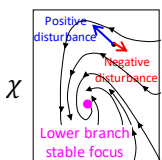

$\Theta / \mathrm{A}$

(b) $A=8.0^{\circ}$ and $f=0.56 \mathrm{~Hz}$ (stable upper branch in the amplitude domain)
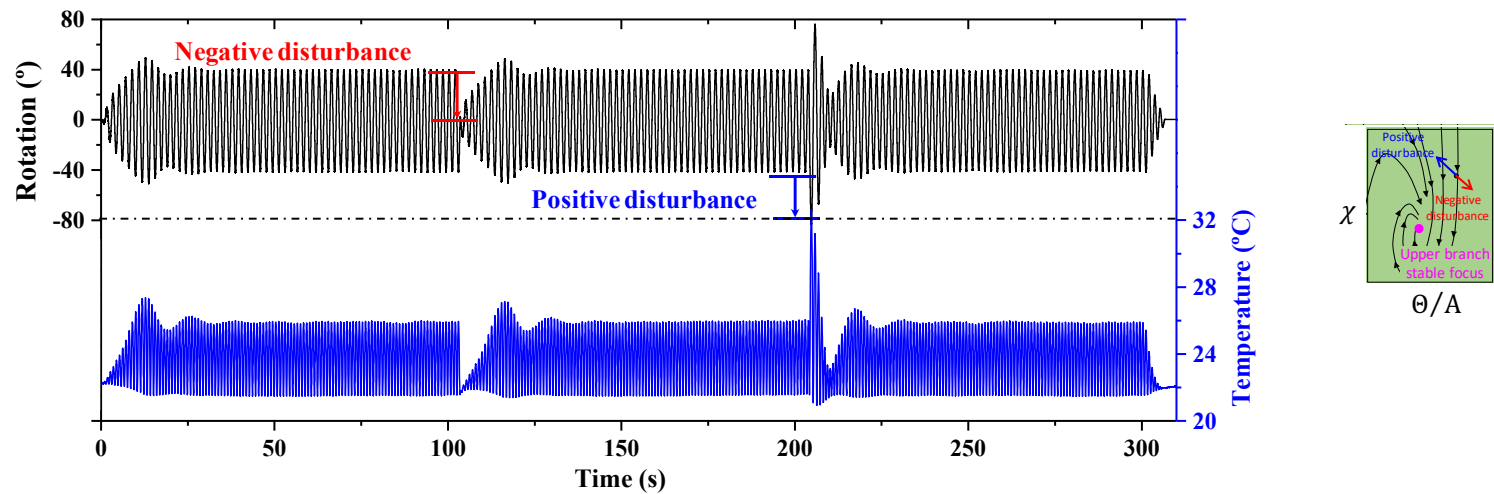

(c) $A=6.6^{\circ}$ and $f=0.56 \mathrm{~Hz}$ (metastable region in the amplitude domain)
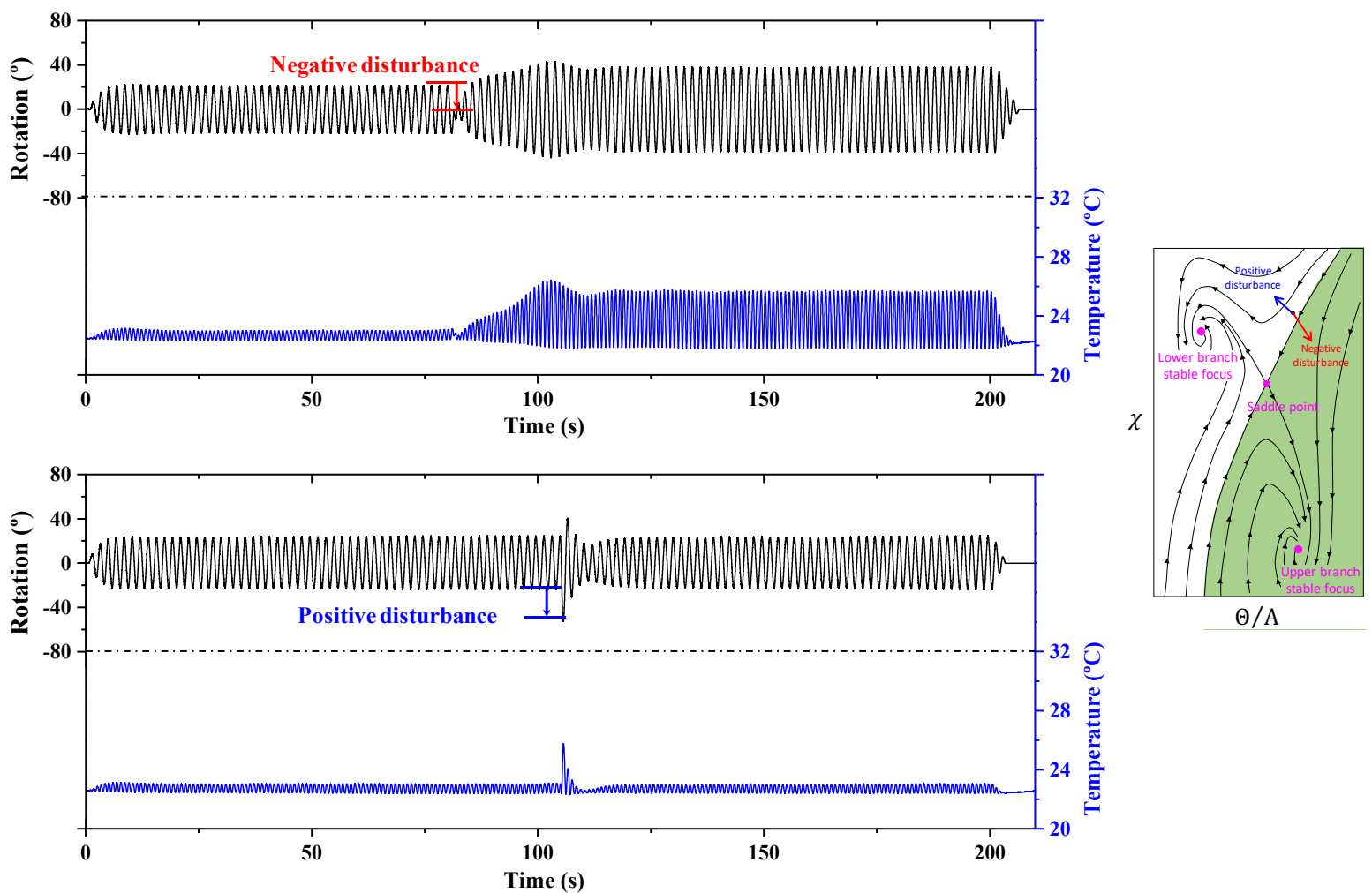

Figure 14. Evolutions of $\theta(t)$ and $T(t)$ at the excitation of $f=0.56 \mathrm{~Hz}$ and (a) $A=6.0^{\circ}$, (b) $A=8.0^{\circ}$, (c) $A=6.6^{\circ}$ with external negative and positive disturbances. 
(a) Negative disturbances at $A=9^{\circ}$ and $f=0.530 \mathrm{~Hz}$ (metastable in the frequency domain)
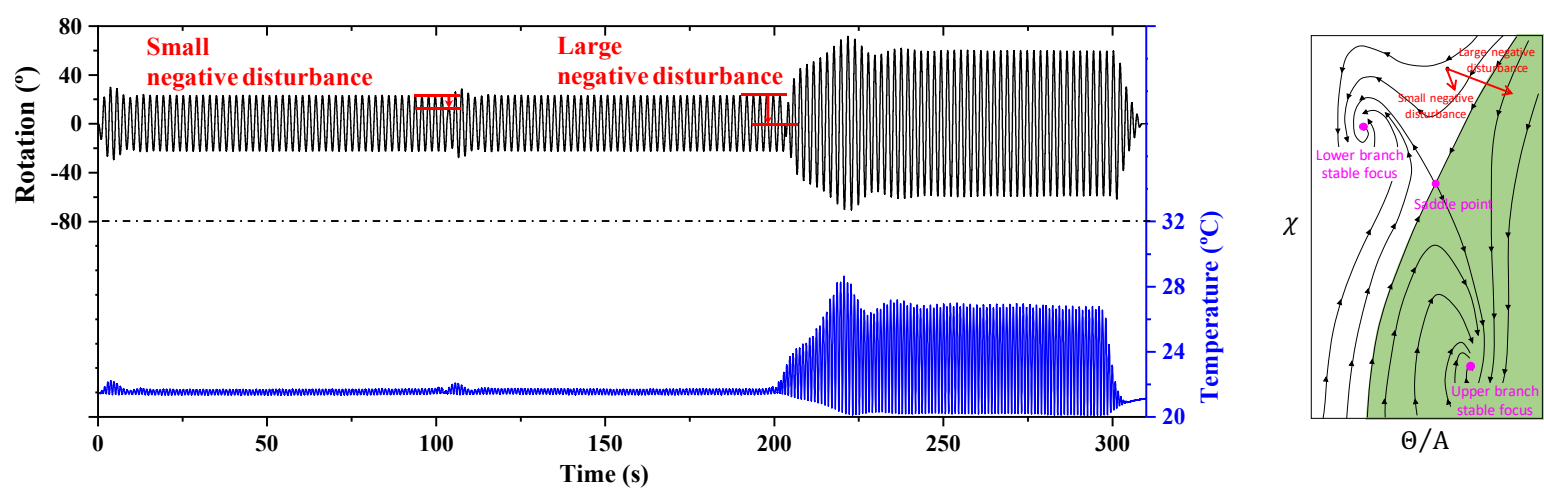

(b) Positive disturbances at $A=9^{\circ}$ and $f=0.530 \mathrm{~Hz}$ (metastable in the frequency domain)
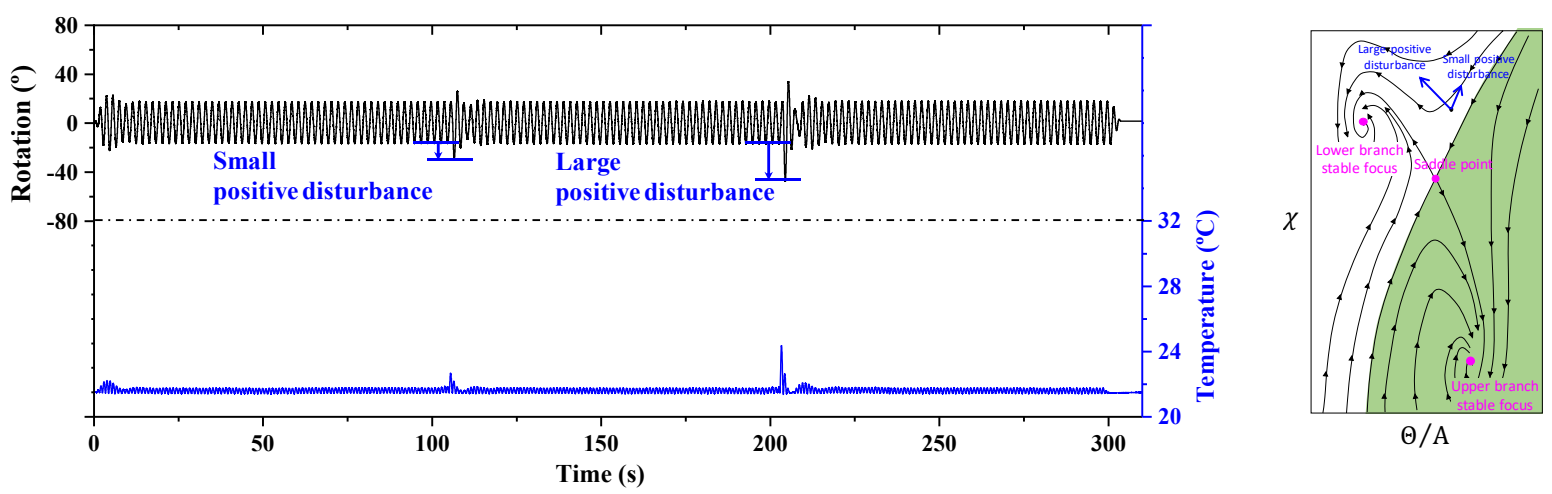

Figure 15. Evolutions of $\theta(t)$ and $T(t)$ at the excitation of $A=9.0^{\circ}$ and $f=0.540 \mathrm{~Hz}$

(a) with small and large negative disturbances; (b) with small and large positive disturbances. 
(a) Negative disturbances at $A=9.2^{\circ}$ and $f=0.54 \mathrm{~Hz}$ (metastable in the amplitude domain)
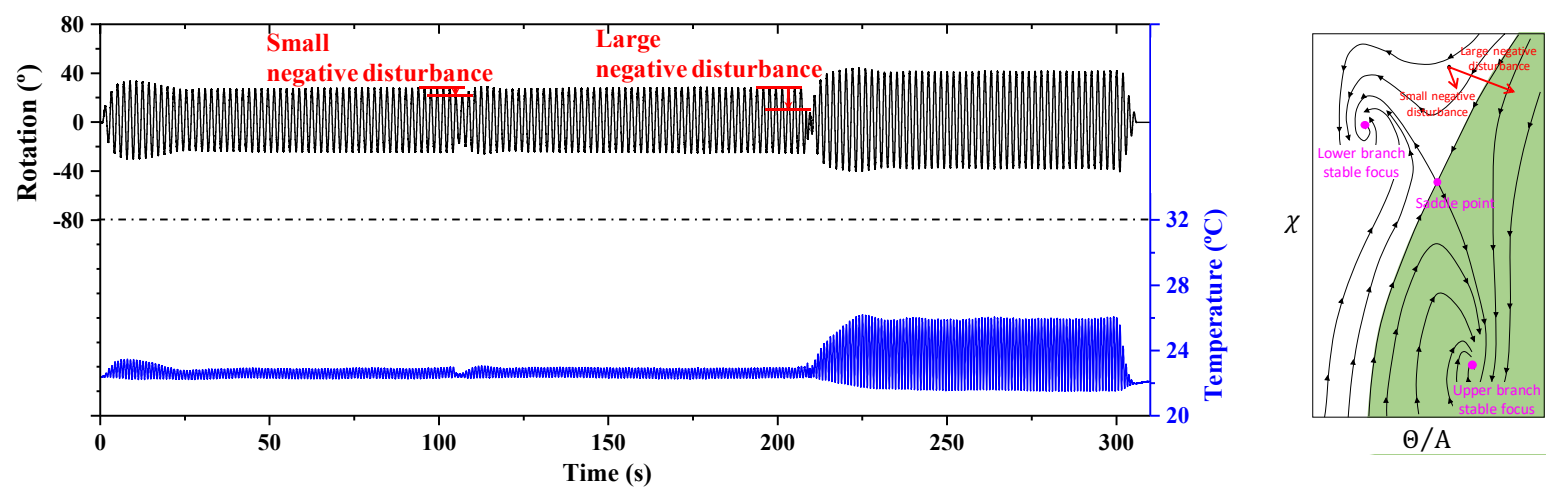

(b) Positive disturbances at $A=9.2^{\circ}$ and $f=0.54 \mathrm{~Hz}$ (metastable in the amplitude domain)
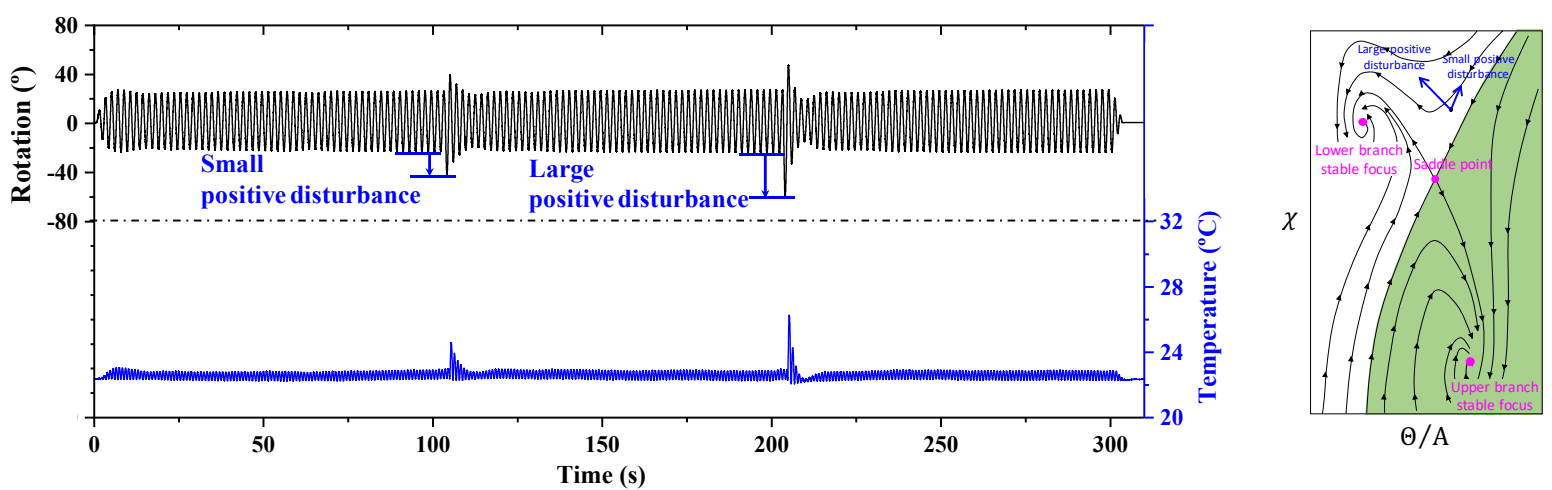

Figure 16. Evolutions of $\theta(t)$ and $T(t)$ at the excitation of $f=0.54 \mathrm{~Hz}$ and $A=9.2^{\circ}$

(a) with small and large negative disturbances; (b) with small and large positive disturbances. 


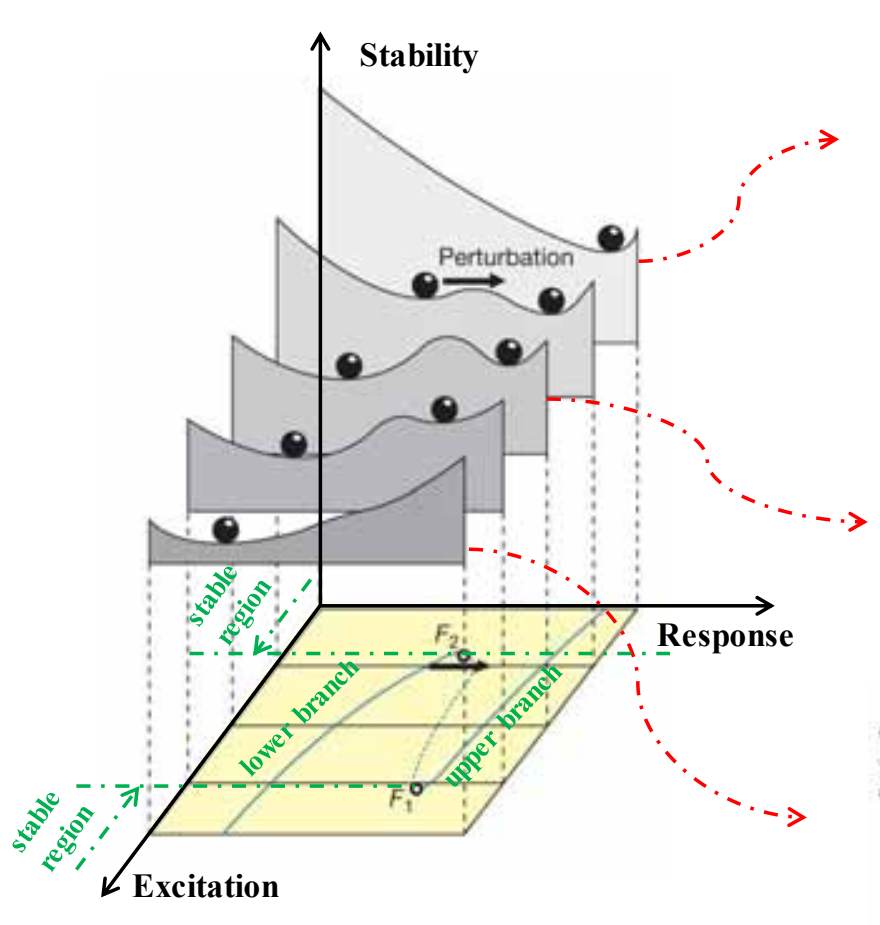

Stable upper branch

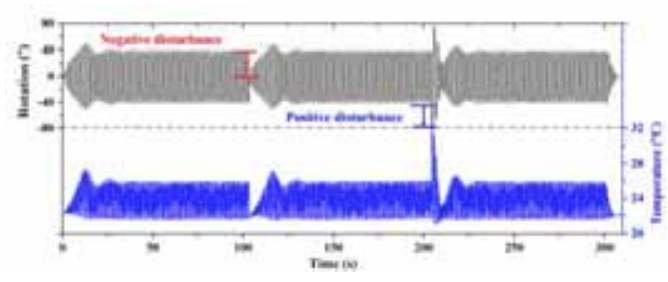

Metastable region

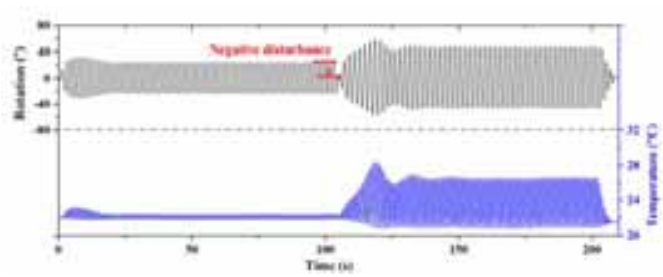

Stable lower branch

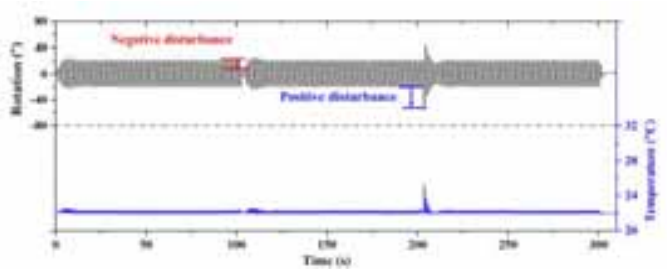

Figure 17. Stability landscape of dynamic response. 